\title{
Spectroscopy and Excited States
}

\author{
Computation of molecular ionization energies using \\ an ensemble density functional theory method \\ Michael Filatov, Seunghoon Lee, and Cheol Ho Choi \\ J. Chem. Theory Comput., Just Accepted Manuscript • Publication Date (Web): 18 May 2020 \\ Downloaded from pubs.acs.org on May 18, 2020
}

\section{Just Accepted}

"Just Accepted" manuscripts have been peer-reviewed and accepted for publication. They are posted online prior to technical editing, formatting for publication and author proofing. The American Chemical Society provides "Just Accepted" as a service to the research community to expedite the dissemination of scientific material as soon as possible after acceptance. "Just Accepted" manuscripts appear in full in PDF format accompanied by an HTML abstract. "Just Accepted" manuscripts have been fully peer reviewed, but should not be considered the official version of record. They are citable by the Digital Object Identifier (DOI®). "Just Accepted" is an optional service offered to authors. Therefore, the "Just Accepted" Web site may not include all articles that will be published in the journal. After a manuscript is technically edited and formatted, it will be removed from the "Just Accepted" Web site and published as an ASAP article. Note that technical editing may introduce minor changes to the manuscript text and/or graphics which could affect content, and all legal disclaimers and ethical guidelines that apply to the journal pertain. ACS cannot be held responsible for errors or consequences arising from the use of information contained in these "Just Accepted" manuscripts. 


\title{
Computation of molecular ionization energies
}

\section{using an ensemble density functional theory}

\section{method}

\author{
Michael Filatov, ${ }^{*,+}$ Seunghoon Lee, ${ }^{\ddagger}$ and Cheol Ho Choi ${ }^{*}+\dagger$ \\ +Department of Chemistry, Kyungpook National University, Daegu 702-701, South Korea \\ $\ddagger$ Division of Chemistry and Chemical Engineering, California Institute of Technology, Pasadena, \\ California 91125, USA \\ E-mail: mike.filatov@gmail.com; cheolho.choi@gmail.com
}

\begin{abstract}
Computation of the ionization energies and of the respective Dyson's orbitals based on the use of the extended Koopmans' theorem (EKT) is implemented in connection with an ensemble density functional theory (eDFT) method, the state-interaction state-averaged spin-restricted ensemble-referenced Kohn-Sham (SI-SA-REKS or SSR) method. The new methodology enables fast computation of the ionization energies and evaluation of the respective Dyson's orbitals, the square norms of which are related with the ionization probabilities, in the ground and excited electronic states of molecules. As the application of EKT recycles the intermediate quantities from the SSR analytical energy gradient, evaluation of the ionization energies and probabilities
\end{abstract}


can be carried out on-the-fly during the non-adiabatic molecular dynamics simulations. This opens up a perspective for fast theoretical simulation of the time-resolved photoelectron spectroscopy observations. In the present work, the new methodology is tested in the computation of the ionization energies and Dyson's orbitals of several molecules in the ground and excited electronic states, including strongly correlated species, such as the ozone molecule, dissociating chemical bonds, and conical intersections.

\section{Introduction}

Time-resolved photoelectron spectroscopy (TRPES) is a powerful pump-probe technique capable of delivering valuable information on the nature of the electronic states along the whole reaction coordinate of a photochemical process. Initially developed to monitor electronic dynamics on surfaces of semiconductors, ${ }^{1}$ TRPES has rapidly become a popular method of investigation of the excited state dynamics in molecules. ${ }^{2}$ In TRPES, a molecule is brought to an excited electronic state by the pump pulse and is subsequently probed by a series of short probe pulses, which cause one-electron ionization of the molecule. ${ }^{2}$ Hence, knowledge of the molecular ionization energies and the respective ionization probabilities covering all possible ionization channels, including the ground and excited electronic states, is required to model TRPES observations theoretically. ${ }^{3}$

The exact description of ionization by a high-frequency electromagnetic radiation is provided by the time-dependent Schrödinger equation. As solution of this equation is not accessible for most practical applications, a number of approximations have been used in the past. Perhaps, the most useful approximation to computing the ionization energies is provided by the Koopmans' theorem, ${ }^{4}$ where the orbital relaxation effects in 
the ionized molecule and the electron correlation in the neutral and the ionized molecules are neglected. A better account of these effects is achieved in the $\triangle \mathrm{SCF}$ approach, ${ }^{5}$ where the ionization energy is computed as a difference between the total energies of the ionized and the neutral molecules. Electron propagator methods ${ }^{6-8}$ enable direct calculation of the ionization energies and allow for certain account of electron correlation effects. A more complete account of the electron correlation effects for the ionization energies is achieved in the GW approximation to the Green's function formalism, ${ }^{9,10}$ which is becoming a standard tool for obtaining quasi-particle energies in the solid state theory. ${ }^{11}$ These methodologies are most commonly used to obtain ionization energies of the ground electronic states of many-electron systems.

The probability of ionization is closely related to the concept of Dyson's orbital, which is defined as an overlap between the wavefunctions of the neutral molecule and of the ion. ${ }^{12,13}$ In the case when an electron is ejected into an unstructured continuum of states, the probability of ionization is proportional to the square norm of the respective Dyson's orbital. ${ }^{3}$ Currently, Dyson's orbitals and the corresponding ionization energies of molecules in the ground and excited electronic states can be calculated with a variety of approaches. ${ }^{14-16}$ However, none of the approaches can be used on-the-fly during the non-adiabatic molecular dynamics (NAMD) simulations. Typically, the Dyson's orbitals are computed retrospectively, using snapshots from a previous NAMD simulation. ${ }^{16}$ This requires an additional computational work and, besides that, the computation of the Dyson's orbitals and of the respective ionization energies are carried out at different levels of approximation. ${ }^{16}$

In this work, a simple method of computing the ionization energies and Dyson's orbitals for the ground and excited electronic states, which can be used during the NAMD simulations, will be presented and tested in a series of molecular calculations. The new 
method is nascent in the spin-restricted ensemble-referenced Kohn-Sham (REKS) method and its state-interaction state-averaged (SI-SA-REKS, or SSR) extension, see Refs. 17 and 18 and references cited therein. The REKS methodology is based on eDFT for ground 19,20 and excited ${ }^{21}$ electronic states and is capable of describing the multi-reference effects arising from near degeneracy of several electronic configurations in the ground and excited (in the SSR variant) states of molecules. The SSR method has the ability to correctly describe the real crossings between the electronic states of the same symmetry, the so-called conical intersections, ${ }^{22}$ including intersections between the ground and excited electronic states. ${ }^{18,23-25}$ Combined with its low computational cost this renders the SSR method suitable for performing the NAMD simulations; a number of successful applications can be found in recent literature. ${ }^{26-32}$

In the SSR method, the Gross-Oliveira-Kohn (GOK) variational principle ${ }^{21}$ for ensembles of the ground and excited electronic states is used in connection with the charge neutral (i.e., the number particle conserving) excitations. Alternatively, an eDFT methodology can be formulated in connection with charged excitations (ionization and/or electron addition), as implemented, e.g., in the N-centered eDFT approach of Senjean and Fromager. ${ }^{33,34}$ In the latter methodology, ${ }^{34}$ the ionization energies can be obtained by differentiating the total ensemble energy with respect to the ensemble weighting factors. As $\mathrm{N}$-centered eDFT also applies to open systems, ${ }^{34}$ this procedure is related with a more traditional formulation of the ionization energies through differentiation with respect to the total number of particles. ${ }^{20,35}$ In the SSR method, the total number of particles is the same in each of the ensemble components and differentiation with respect to the ensemble weights yields the electronic excitation energies, rather than the ionization energies. Differentiation with respect to the total number of particles is not defined in the SSR method 
either and, for obtaining the ionization energies, one needs to follow a different route than, e.g., in the N-centered eDFT methodology.

In the present work, to calculate the ionization energies of the individual states in the SSR method, we propose to use the extended Koopmans' theorem (EKT). ${ }^{36}$ EKT enables the exact computation of the ionization energies of a many-particle system ${ }^{37,38}$ through the eigenvalues of the Lagrangian expressed in terms of the natural orbitals. ${ }^{36,39}$ As the relaxed density matrix and the Lagrangian for the SSR individual states are available during the analytic gradient computation, ${ }^{40}$ the ionization energies in the EKT-SSR method can be obtained during the NAMD simulations, at no additional cost. In the following, the key aspects of the EKT-SSR method will be briefly outlined before describing its application to the ionization energies of molecules in the ground and excited states; including strongly correlated systems, such as the ozone molecule, dissociating chemical bonds, and conical intersections between the ground and excited electronic states.

\section{Methodology}

The SSR method has been described in the literature; ${ }^{17,18,23,41,42}$ hence, only a brief account of the method will be given here. The SSR method is based on EDFT, where ground state $\operatorname{eDFT}^{19,20}$ is used to describe the non-dynamic electron correlation originating in multireference ground states of molecules and eDFT for ensembles of the ground and excited states ${ }^{21}$ to obtain excitation energies from a variational time-independent computation. The ensemble representation of the density and the energy of a multi-reference electronic state results in the fractional occupation numbers (FONs) of several frontier KS orbitals. ${ }^{43-45}$ 
Currently, the SSR method is available for systems with two fractionally occupied KS orbitals ${ }^{17,18,41,42}$ and four fractionally occupied KS orbitals; ${ }^{46-48}$ this is sufficient to describe the dissociation of a single and a double bond, respectively.* In the case of two fractionally occupied KS orbitals accommodating in total two electrons, i.e., the $(2,2)$ active space, the ground electronic state can be approximated by a perfectly spin-paired singlet (PPS) electronic configuration and an excited state by an open-shell singlet (OSS) configuration. ${ }^{46}$ For both configurations, the energy expression can be written as ${ }^{46}$

$$
E^{X}=\sum_{L=1}^{L_{\max }} C_{L}^{X} E\left[\rho_{L}^{\alpha}, \rho_{L}^{\beta}\right] ; \quad \sum_{L=1}^{L_{\max }} C_{L}^{X}=1
$$

where $X=P P S, O S S$ and the coefficients $C_{L}^{X}$ depend on the fractional occupation numbers of the active orbitals. The densities of the microstates $(\sigma=\alpha, \beta$ denotes the spin)

$$
\rho_{L}^{\sigma}(\mathbf{r})=\sum_{q}^{o c c} n_{q, L}^{\sigma}\left|\varphi_{q}(\mathbf{r})\right|^{2}
$$

are calculated using a common set of orbitals $\varphi_{q}(\mathbf{r})$ and the integer and fixed occupation numbers $n_{q, L}^{\sigma}$ defined for each microstate. ${ }^{17,18,41}$ For the OSS state, the $C_{L}^{O S S}$ coefficients are fixed by spin-symmetry and, for the PPS state, the $C_{L}^{P P S}$ coefficients are obtained by variational optimization of the respective FONs; see Refs. 41, 17, 18, and 40 for more detail.

\footnotetext{
${ }^{*}$ Further extension of the methodology to larger active spaces is possible along the guidelines outlined
} in Ref. 46. 
In the SA-REKS method, ${ }^{42}$ an ensemble of the PPS and OSS states ${ }^{\dagger}$

$$
\begin{aligned}
E^{S A-R E K S}= & w_{P P S} E_{0}^{P P S}+w_{O S S} E_{1}^{O S S} \\
& w_{P P S}+w_{O S S}=1
\end{aligned}
$$

is optimized with respect to the FONs and the KS orbitals; the latter are subject to the orthonormality constraints. This results in a set of one-electron equations

$$
f_{q} \hat{F}_{q} \varphi_{q}(\mathbf{r})=\sum_{p}^{o c c} \varphi_{p}(\mathbf{r}) \varepsilon_{p q}
$$

where $f_{q}=n_{q} / 2$ is the average occupation number of the $q$-th spin-orbital, $\varepsilon_{p q}$ are the Lagrange multipliers for the orthonormality constraints, the one-electron Fock operators $\hat{F}_{q}$ are defined as

$$
\hat{F}_{q}=\frac{1}{2 f_{q}} \sum_{L}^{L_{\max }} C_{L}^{S A} \sum_{\sigma} n_{q, L}^{\sigma} \hat{F}_{L}^{\sigma}
$$

with the Fock operators $\hat{F}_{L}^{\sigma}$ of the individual microstates given by

$$
\hat{F}_{L}^{\sigma}=\hat{h}+\sum_{q} \sum_{\sigma} n_{q, L}^{\sigma} \int \frac{\varphi_{q}^{*}\left(\mathbf{r}^{\prime}\right) \varphi_{q}\left(\mathbf{r}^{\prime}\right)}{\left|\mathbf{r}-\mathbf{r}^{\prime}\right|} d \mathbf{r}^{\prime}+V_{x c, L}^{\sigma}(\mathbf{r})
$$

Eq. (4) is solved by the use of the coupling operator technique, ${ }^{50}$ where the matrix of the coupling operator

$$
\hat{F}_{q p}=\frac{f_{q} \hat{F}_{q}-f_{p} \hat{F}_{p}}{f_{q}-f_{p}}
$$

\footnotetext{
†The subscripts 0 and 1 are added to underline that the PPS state is regarded as the ground state and the OSS state as an excited state. Here, the ensemble energy is formulated as an explicit sum of the energies of the individual states. An alternative formulation of the $\mathrm{GOK}^{21}$ ensemble formalism operates with a weight-dependent ensemble XC functional. ${ }^{49}$ In the latter case, the energies of the individual states can be extracted by differentiating the ensemble energy with respect to the ensemble weights. ${ }^{49}$
} 
is repeatedly diagonalized until the convergence (of the energy and the density matrix) is reached. When the orbitals are converged, the following conditions are fulfilled

$$
\forall p, q ; \quad \varepsilon_{p q}=\varepsilon_{q p} \quad \Longrightarrow \quad\left\langle\varphi_{p}\left|f_{q} \hat{F}_{q}\right| \varphi_{q}\right\rangle=\left\langle\varphi_{q}\left|f_{p} \hat{F}_{p}\right| \varphi_{p}\right\rangle
$$

for the elements of the Lagrangian matrix in Eq. (4); ${ }^{50}$ however the off-diagonal Lagrange multipliers between the orbitals with different occupations do not vanish. ${ }^{\ddagger}$ Generally, the Lagrange matrix in open-shell SCF methodologies does not become diagonal upon convergence and the eigenfunctions of Eq. (4) are not the canonical orbitals satisfying the Koopmans' theorem. ${ }^{4}$

In the SSR $(2,2)$ method, ${ }^{23}$ the variational optimization of the KS orbitals and their FONs is followed by solving a simple $2 \times 2$ secular problem

$$
\left(\begin{array}{cc}
E_{0}^{P P S} & \Delta_{01}^{S A} \\
\Delta_{01}^{S A} & E_{1}^{O S S}
\end{array}\right)\left(\begin{array}{ll}
a_{00} & a_{01} \\
a_{10} & a_{11}
\end{array}\right)=\left(\begin{array}{cc}
E_{0} & 0 \\
0 & E_{1}
\end{array}\right)\left(\begin{array}{ll}
a_{00} & a_{01} \\
a_{10} & a_{11}
\end{array}\right)
$$

to include possible coupling between the PPS and the OSS electronic configurations. In Eq. (9), $E_{0}^{P P S}$ and $E_{1}^{O S S}$ are the energies obtained in the SA-REKS $(2,2)$ orbital optimization and the interstate coupling element $\Delta_{01}^{S A}$ is calculated using the SA-REKS $(2,2)$ Lagrangian matrix element $\varepsilon_{a b}^{S A}$ between the active orbitals $\phi_{a}$ and $\phi_{b}$ as

$$
\Delta_{01}^{S A}=\left(\sqrt{n_{a}}-\sqrt{n_{b}}\right) \varepsilon_{a b}^{S A}
$$

\footnotetext{
¥The occurrence of the off-diagonal Lagrange multipliers in the open-shell SCF methods is the consequence of the variational principle ${ }^{50}$ and does not mean that an extra correlation energy is accounted for in this way. In the REKS method, the total energy of the $E_{0}^{P P S}$ and the $E_{1}^{O S S}$ configurations is calculated using Eq. (1), where only the KS orbitals and not the Lagrange multipliers nor the Fock matrix (6) elements are used. Hence, no double counting of the correlation energy occurs due to the off-diagonal Lagrange multipliers. More detail on the absence of the double counting in the REKS-type methods can be found in Refs. 17 and 18.
} 
where $n_{a}$ and $n_{b}$ are the FONs of the active orbitals. ${ }^{18,23,46}$

The analytical energy derivatives of the SSR individual states with respect to an external perturbation $\lambda$ (e.g., nuclear displacement $)^{40}$ can be represented as

$$
\begin{aligned}
\frac{\partial E_{X}}{\partial \lambda} & =\operatorname{tr} \mathbf{D}^{X} \mathbf{h}^{\lambda}-\frac{1}{2} \operatorname{tr} \tilde{\mathbf{W}}^{X} \mathbf{S}^{\lambda} \\
& +\sum_{L} \tilde{C}_{L}^{X} \frac{\partial^{\prime} E_{L}^{2 e}}{\partial \lambda}-\sum_{L} C_{L}^{S A} \sum_{\sigma} \operatorname{tr}^{X} \mathbf{R}_{L}^{\sigma} \mathbf{T}_{L}^{\sigma, \lambda(2 e)}
\end{aligned}
$$

where $X=0,1$ labels the SSR state, $\mathbf{D}^{X}$ is the relaxed density matrix of the state $X$, and $\tilde{\mathbf{W}}^{X}$ is the effective Lagrangian matrix in the basis of the eigenfunctions of Eq. (4)

$$
\tilde{W}_{p q}^{X}=\sum_{L} \tilde{C}_{L}^{X}\left({ }^{p} \epsilon_{p q}^{L}+{ }^{q} \epsilon_{p q}^{L}\right)-2\left({ }^{X} Q_{p q}^{(1)}+{ }^{X} Q_{p q}^{(2)}\right)
$$

where the ${ }^{j} \epsilon_{p q}^{L}$ coefficients are given by

$$
{ }^{j} \epsilon_{p q}^{L}=\sum_{\sigma}\left\langle p\left|n_{j, L}^{\sigma} \hat{F}_{L}^{\sigma}\right| q\right\rangle
$$

In eq. (11b), the terms $\partial^{\prime} E_{L}^{2 e} / \partial \lambda$ and $\mathbf{T}_{L}^{\sigma, \lambda(2 e)}$ contain the derivatives of the two-electron integrals $\S$ and of the exchange-correlation potential; their expressions can be found in Ref. 40. The modified ensemble weighting factors $\tilde{C}_{L}^{X}$, the matrices ${ }^{X} \mathbf{R}_{L}^{\sigma}, \mathbf{Q}^{(1)}$, and $\mathbf{Q}^{(2)}$ in Eqs. (11b) and (12) depend on the response vector $\mathbf{Z}$ obtained from solving the coupledperturbed (CP) REKS equations; see Ref. 40 for detail.

In the basis of the eigenfunctions of the SA-REKS one-electron equations (4), the re-

\footnotetext{
$\S$ Prime in the $\partial^{\prime} / \partial \lambda$ symbol means that only the integrals need to be differentiated and not the density matrix elements
} 
laxed density matrix $\mathbf{D}^{X}$ is given by

$$
\begin{aligned}
D_{q p}^{X} & =\delta_{q p} \sum_{L} \tilde{C}_{L}^{X}\left(n_{p, L}^{\alpha}+n_{p, L}^{\beta}\right)-2 Z_{q p}^{X}\left(f_{p}^{S A}-f_{q}^{S A}\right) \\
& -4 \delta_{q b} \delta_{p a} a_{0 X} a_{1 X}\left(\sqrt{n_{a}} f_{a}^{S A}-\sqrt{n_{b}} f_{b}^{S A}\right)
\end{aligned}
$$

where $Z_{i j}^{X}$ is the $Z$-vector obtained from solving the CP-REKS equations for the state $X$ $(=0,1), a_{k j}$ are the elements of the eigenvectors of the SSR secular equation (9), $n_{a}$ and $n_{b}$ are the FONs of the active orbitals in the PPS configuration, and $f_{j}^{S A}$ are the FONs of the SA-REKS orbitals in the averaged state. The matrix $\mathbf{D}^{X}$ is the proper one-electron density matrix for the SSR individual state $X$ and it yields the first order properties, e.g., the dipole moment, by taking the trace of its product with the matrix of the respective one-electron operator. ${ }^{51}$

As seen in Eq. (11a), the matrix $\tilde{\mathbf{W}}^{X}$ gives the contribution to the gradient of the derivative of the orbital orthonormality constraint, i.e., the Pulay term. ${ }^{52}$ Hence, this matrix is identified with the (effective) Lagrangian matrix for the individual state $X$; this is similar with the SA-CASSCF methodology. ${ }^{53}$ If the energy derivative in Eq. (11) was calculated for a single state (e.g., by setting $w_{P P S}=1$ ) obtained self-consistently from solving the secular equation (4), then the response vector $\mathbf{Z}$ would vanish and the relaxed density matrix in Eq. (11) would become identical to the one-particle density matrix computed from the eigenfunctions of Eq. (4) and the Lagrangian in Eq. (11) would become identical to the Lagrangian in Eq. (4); this would recover the usual analytic gradient of a variationally obtained energy. ${ }^{52}$

Although it is not possible to obtain the canonical orbitals for an individual state in the SSR method, the ionization energies and the respective one-electron functions (Dyson's 
orbitals) can be obtained from EKT. ${ }^{36}$ EKT states that the (negatives of the) ionization energies and Dyson's orbitals span the eigensystem of the Lagrangian matrix expressed in the basis of the natural orbitals; ${ }^{36}$ this symmetric eigenproblem can be conveniently reformulated as a generalized eigenproblem ${ }^{54}$ for the $\left[\tilde{\mathbf{W}}^{X}, \mathbf{D}^{X}\right]$ matrix pair. ${ }^{39}$ In the case of the SSR method, this yields

$$
\tilde{\mathbf{W}}^{X} \mathbf{C}^{X}=\mathbf{D}^{X} \mathbf{C}^{X} \varepsilon^{X}
$$

where $\varepsilon^{X}$ are the (negative) ionization energies for the individual state $X$ and $C^{X}$ are the respective Dyson's orbitals. Obviously, the eigenfunctions $\mathbf{C}^{X}$ are not normalized on the space metric and the square of their norm

$$
\|\gamma\|^{2}=\operatorname{tr}\left(\left(\mathbf{C}^{X}\right)^{\dagger} \mathbf{D}^{X}\left(\mathbf{D}^{X}\right)^{\dagger} \mathbf{C}^{X}\right)
$$

yields the pole strength of the respective ionization. ${ }^{39}$ The Dyson's norms (16) yield a rough approximation to the ionization probability, when a transition of the ionized electron occurs into unstructured continuum states. ${ }^{3}$

Before applying Eq. (15) in practical calculations, a subtle issue needs to be addressed. Generally, the natural orbital occupation numbers (the eigenvalues of the density matrix) do not vanish for all the natural orbitals. ${ }^{55}$ However, they may become very small and make the density matrix nearly singular. ${ }^{39}$ As the density matrix occurs on the right hand side of the EKT equation (15), its near singularity makes application of the standard methods of solving generalized eigenvalue problems ${ }^{54}$ problematic. ${ }^{\llbracket}$ One way to circumvent this difficulty is to drop all the eigenvalues of the relaxed density matrix less than a certain

\footnotetext{
INote that the near singularity of the density matrix has nothing to do with the linear dependencies in the one-particle basis set; which leads to (near) singularity of the overlap matrix.
} 
threshold value $\left(10^{-6}\right.$ in this work) and to reconstruct both the Lagrangian $\tilde{\mathbf{W}}^{X}$ and the density matrix $\mathbf{D}^{X}$ using the remaining natural orbitals and their occupation numbers. ${ }^{39}$ Then, the secular problem (15) is solved with the new metric $\mathbf{d}^{X}$ in the usual way using symmetric orthogonalization of the left hand side. ${ }^{39}$

The described approach is easy to implement once the analytic energy gradient is available. The EKT formalism was previously used in connection with ab initio correlated methods. ${ }^{39,56}$ As for the TDDFT and SF-TDDFT methods the gradients are available, the EKT formalism can be used in connection with these methods as well. Presently, an EKT extension of the (mixed reference SF) MRSF-TDDFT method, ${ }^{57}$ where the erroneous spincontamination of SF-TDDFT is eliminated, however not at the expense of its ability to describe strongly correlated ground and excited states, is under construction.

All the calculations reported here were carried out using a locally modified version of the GAMESS-US program. ${ }^{58}$ All the calculations employ the $6-311 \mathrm{G}^{* *}$ basis set ${ }^{59}$ and the BH\&HLYP density functional, ${ }^{60}$ unless noted otherwise. When solving the SA-REKS SCF equations (4), the $w_{P P S}$ weighting factor was set to its default value of 0.5. ${ }^{42}$

\section{Results and Discussion}

Here, the described EKT-SSR formalism will be applied to the calculation of the ionization energies of a number of molecules, where the formalism will be first validated for a set of molecules, the ground states of which are well described within the standard single determinant approximation, and then applied to the excited states and to molecules with strongly correlated ground state, e.g., ozone ${ }^{61-63}$ and dissociating single bonds in $\mathrm{H}_{2}$ and $\mathrm{LiH}$. In the latter case, it is expected that the norms of the Dyson's orbitals $|\gamma|^{2}$ may 
noticeably deviate from unity. Indeed, Dyson's orbital for a specific ionization channel is defined as the overlap of the parent many-electron wavefunction of the neutral molecule with the wavefunction of the ionized molecule. ${ }^{12,13}$ In the simplest case of single bond dissociation, e.g., $\mathrm{H}_{2}$, the ground state wavefunction can be approximated by a PPS configuration

$$
\Phi_{P P S}=\sqrt{\frac{n_{a}}{2}}\left|\ldots \phi_{a} \bar{\phi}_{a}\right\rangle-\sqrt{\frac{n_{b}}{2}}\left|\ldots \phi_{b} \bar{\phi}_{b}\right\rangle
$$

where $\phi_{a}$ and $\phi_{b}$ are the bonding and the antibonding orbitals, respectively, and $n_{a}$ and $n_{b}$ are their FONs. Assuming that the ground state of the ionized molecule is approximated by a single determinant configuration $\left|\ldots \phi_{a}^{\prime}\right\rangle$, where $\phi_{a}^{\prime}$ is the singly occupied orbital of the ion, the Dyson's orbital $\Phi_{D}$ is approximated by

$$
\Phi_{D} \approx \sqrt{\frac{n_{a}}{2}}\left\langle\ldots \phi_{a} \bar{\phi}_{a} \mid \ldots \phi_{a}^{\prime}\right\rangle-\sqrt{\frac{n_{b}}{2}}\left\langle\ldots \phi_{b} \bar{\phi}_{b} \mid \ldots \phi_{a}^{\prime}\right\rangle
$$

where the integrals in the brackets are taken over the $N-1$ electrons in the ionized molecule. In the limiting case of $\phi_{a} \approx \phi_{a}^{\prime}$, Eq. (18) yields $\left|\gamma_{D}\right|^{2} \approx n_{a} / 2$, which becomes fractional for fractional $n_{a}$. Hence, besides the overlap between the orbitals of the neutral and the ionized molecule, the norm of the Dyson's orbital and the probability of ionization ${ }^{3}$ are defined by the FONs of the frontier orbitals in the multi-reference state. In the case of $n_{a}$ and $n_{b}$ strongly deviating from 2 and 0 (the FONs 2 and 0 correspond to a single determinant wavefunction), the occurrence of fractionally normalized Dyson's orbitals resembling the orbitals $\phi_{a}$ and $\phi_{b}$ may be expected. The same reasoning can be applied to the OSS configuration approximating an excited state of the simple system discussed 
above. As the OSS configuration

$$
\Phi_{1}=\frac{1}{\sqrt{2}}\left|\ldots \phi_{a} \bar{\phi}_{b}\right\rangle+\frac{1}{\sqrt{2}}\left|\ldots \phi_{b} \bar{\phi}_{a}\right\rangle
$$

combines two single determinants with equal weights, it may be expected that two Dyson's orbitals with (nearly) equal fractional norms occur in the ionization spectrum of the molecule. Although the arguments presented above may seem self-evident and they have been discussed in the literature, ${ }^{3,64}$ it seems nevertheless useful to reiterate them before discussing the results obtained in this work.

\subsection{Validation of EKT-SSR ionization energies}

The lowest ionization energies of several closed-shell molecules selected from compilation by Chong et al. ${ }^{65}$ were computed using the EKT-SSR method. The molecules, see Table 1 , were selected by the criterion that their electronic structure can be well represented by the SSR $(2,2)$ method; i.e., the excited state included into the state averaging is well represented by the $\mathrm{HOMO} \rightarrow \mathrm{LUMO}$ one-electron transition and the HOMO and the LUMO are non-degenerate. The EKT-SSR calculations employed the $6-311 G^{* *}$ basis set $^{59}$ and two density functionals, the BH\&HLYP hybrid functional ${ }^{60}$ and the HF exchange-only (X-only) functional. The latter functional was used as it is self-interaction error (SIE) free and yields the correct long-range behavior of the one-electron potential; which is crucial for obtaining correct ionization energies. ${ }^{36,66}$ Although the standard GGA and the hybrid exchange-correlation (XC) energy functionals, such as the BH\&HLYP functional, do not yield the correct $1 / r$ decay of the XC potential and produce large errors (on the order of 1 $\mathrm{eV}$ or more) in the valence ionization energies estimated by the Koopmans' theorem, the 
deviation from the experimental values appears to be systematic ${ }^{65,67}$ and can be largely corrected by shifting the smallest ionization energy to match the first ionization potential of the molecule; ${ }^{65}$ no such a shift is required for the ionization energies obtained with the use of the HF X-only functional.

Table 1: Ionization energies of several closed-shell molecules computed using the EKTSSR method and the standard Koopmans' theorem for the RKS/RHF method. The reference experimental energies are taken from compilation by Chong et al. ${ }^{65}$

\begin{tabular}{|c|c|c|c|c|c|c|c|c|}
\hline \multirow{2}{*}{ Molecule } & \multirow{2}{*}{ Symm. ${ }^{a)}$} & \multirow{2}{*}{ Expt. } & \multicolumn{2}{|c|}{ EKT-SSR-BH\&HLYP } & \multirow{2}{*}{ RBH\&HLYP } & \multicolumn{2}{|c|}{ EKT-SSR-HF } & \multirow{2}{*}{ RHF } \\
\hline & & & $\mathrm{IE}^{\mathrm{b})}$ & norm $^{c)}$ & & IE & norm & \\
\hline \multirow[t]{2}{*}{$\mathrm{HF}$} & $1 \pi$ & 16.19 & 13.53 (16.19) & 1.00 & 13.47 (16.19) & 17.43 & 1.00 & 17.34 \\
\hline & $3 \sigma$ & 19.90 & $16.73(19.39)$ & 0.99 & $17.05(19.76)$ & 22.71 & 0.84 & 20.53 \\
\hline \multirow[t]{3}{*}{$\mathrm{HCl}$} & $2 \pi$ & 12.77 & 10.76 (12.77) & 1.00 & 10.76 (12.77) & 12.95 & 1.00 & 12.93 \\
\hline & $5 \sigma$ & 16.60 & 14.59 (16.60) & 1.00 & $14.71(16.72)$ & 17.30 & 0.97 & 17.01 \\
\hline & $4 \sigma$ & 25.80 & $26.16(28.17)$ & 1.00 & $26.12(28.13)$ & 30.46 & 1.00 & 30.41 \\
\hline \multirow[t]{5}{*}{$\mathrm{H}_{2} \mathrm{CO}$} & $2 b_{2}$ & 10.90 & $9.25(10.90)$ & 1.00 & $9.32(10.90)$ & 11.77 & 0.99 & 11.93 \\
\hline & $1 b_{1}$ & 14.50 & $12.65(14.30)$ & 0.99 & $12.54(14.12)$ & 14.61 & 0.99 & 14.50 \\
\hline & $5 a_{1}$ & 16.10 & $14.61(16.26)$ & 1.00 & $14.60(16.18)$ & 17.74 & 1.00 & 17.66 \\
\hline & $1 b_{2}$ & 17.00 & $15.76(17.41)$ & 1.00 & $15.75(17.33)$ & 18.69 & 1.00 & 18.77 \\
\hline & $4 a_{1}$ & 21.40 & $19.92(21.57)$ & 1.00 & $19.89(21.47)$ & 23.54 & 1.00 & 23.53 \\
\hline \multirow[t]{5}{*}{$\mathrm{H}_{2} \mathrm{CS}$} & $3 b_{2}$ & 9.38 & $7.76(9.38)$ & 1.00 & $7.80(9.38)$ & 9.46 & 1.00 & 9.53 \\
\hline & $2 b_{1}$ & 11.76 & $10.22(11.85)$ & 0.98 & $10.03(11.61)$ & 11.57 & 0.98 & 11.34 \\
\hline & $7 a_{1}$ & 13.85 & 12.55 (14.18) & 1.00 & $12.53(14.11)$ & 14.74 & 1.00 & 14.70 \\
\hline & $2 b_{2}$ & 15.20 & $14.78(16.40)$ & 1.00 & $14.83(16.41)$ & 17.45 & 1.00 & 17.56 \\
\hline & $6 \mathrm{a}_{1}$ & 19.90 & $18.84(20.47)$ & 1.00 & $18.86(20.44)$ & 22.10 & 1.00 & 22.13 \\
\hline \multirow[t]{6}{*}{$\mathrm{C}_{2} \mathrm{H}_{4}$} & $1 b_{3 u}$ & 10.68 & $8.63(10.68)$ & 1.00 & 8.71 (10.68) & 10.11 & 0.99 & 10.17 \\
\hline & $1 b_{3 g}$ & 12.80 & $11.48(13.53)$ & 1.00 & $11.48(13.45)$ & 13.75 & 1.00 & 13.77 \\
\hline & $3 a_{g}$ & 14.80 & $13.32(15.36)$ & 1.00 & $13.32(15.29)$ & 15.84 & 1.00 & 15.86 \\
\hline & $1 \mathrm{~b}_{2 u}^{\circ}$ & 16.00 & $14.78(16.83)$ & 1.00 & 14.79 (16.76) & 17.42 & 1.00 & 17.44 \\
\hline & $2 \mathrm{~b}_{1 u}$ & 19.10 & $18.16(20.20)$ & 1.00 & $18.16(20.13)$ & 21.46 & 1.00 & 21.48 \\
\hline & $2 \mathrm{a}_{g}$ & 23.60 & $23.67(25.72)$ & 1.00 & $23.67(25.64)$ & 28.01 & 1.00 & 28.03 \\
\hline \multirow[t]{11}{*}{ furan } & $1 a_{2}$ & 9.00 & $7.38(9.00)$ & 1.00 & $7.45(9.00)$ & 8.48 & 1.00 & 8.65 \\
\hline & $2 b_{1}$ & 10.40 & $9.13(10.75)$ & 1.00 & $9.07(10.62)$ & 11.01 & 0.97 & 10.76 \\
\hline & $9 a_{1}$ & 13.00 & $12.12(13.74)$ & 1.00 & $12.13(13.69)$ & 14.49 & 1.00 & 14.61 \\
\hline & $8 a_{1}$ & 13.80 & $12.73(14.35)$ & 1.00 & $12.73(14.28)$ & 15.43 & 1.00 & 15.34 \\
\hline & $6 b_{2}$ & 14.40 & 13.18 (14.80) & 1.00 & $13.17(14.72)$ & 15.52 & 1.00 & 15.70 \\
\hline & $5 b_{2}$ & 15.25 & 14.05 (15.67) & 1.00 & $14.06(15.61)$ & 16.60 & 1.00 & 16.57 \\
\hline & $1 b_{1}$ & 15.60 & $14.30(15.92)$ & 1.00 & $14.32(15.87)$ & 18.15 & 0.95 & 17.14 \\
\hline & $7 a_{1}$ & 17.50 & $17.04(18.66)$ & 1.00 & $17.04(18.59)$ & 20.10 & 1.00 & 20.09 \\
\hline & $6 a_{1}$ & 18.80 & 17.93 (19.55) & 1.00 & $17.92(19.48)$ & 21.19 & 1.00 & 21.26 \\
\hline & $4 b_{2}$ & 19.70 & $18.65(20.27)$ & 1.00 & $18.66(20.21)$ & 21.86 & 1.00 & 21.90 \\
\hline & $3 b_{2}$ & 23.00 & $23.15(24.77)$ & 1.00 & $23.15(24.71)$ & 27.20 & 1.00 & 27.28 \\
\hline \multirow[t]{4}{*}{ thiophene } & $1 \mathrm{a}_{2}$ & 8.87 & $7.58(8.87)$ & 1.00 & $7.66(8.87)$ & 8.78 & 1.00 & 8.89 \\
\hline & $3 b_{1}$ & 9.52 & $8.13(9.42)$ & 0.99 & $8.06(9.27)$ & 9.43 & 1.00 & 9.38 \\
\hline & $11 \mathrm{a}_{1}$ & 12.10 & $10.95(12.24)$ & 1.00 & $10.96(12.17)$ & 12.90 & 1.00 & 12.91 \\
\hline & $2 b_{1}$ & 12.70 & 11.98 (13.27) & 1.00 & $11.99(13.20)$ & 14.10 & 1.00 & 14.11 \\
\hline
\end{tabular}


Table 1 - Continued from previous page

\begin{tabular}{|c|c|c|c|c|c|c|c|c|}
\hline \multirow{2}{*}{ Molecule } & \multirow{2}{*}{ Symm. } & \multirow{2}{*}{ Expt. } & \multicolumn{2}{|c|}{ EKT-SSR-BH\&HLYP } & \multirow{2}{*}{ RBH\&HLYP } & \multicolumn{2}{|c|}{ EKT-SSR-HF } & \multirow{2}{*}{ RHF } \\
\hline & & & IE & norm & & IE & norm & \\
\hline & $7 \mathrm{~b}_{2}$ & 13.30 & $12.23(13.53)$ & 1.00 & $12.23(13.44)$ & 14.33 & 1.00 & 14.32 \\
\hline & $10 \mathrm{a}_{1}$ & 13.90 & $12.52(13.82)$ & 1.00 & $12.51(13.72)$ & 14.91 & 1.00 & 14.90 \\
\hline & $6 b_{2}$ & 14.30 & $13.17(14.46)$ & 1.00 & $13.16(14.37)$ & 15.68 & 1.00 & 15.67 \\
\hline & $9 a_{1}$ & 16.60 & $16.12(17.41)$ & 1.00 & $16.11(17.32)$ & 18.92 & 1.00 & 18.91 \\
\hline & $5 b_{2}$ & 17.60 & $17.31(18.60)$ & 1.00 & $17.30(18.51)$ & 20.39 & 1.00 & 20.38 \\
\hline & $8 a_{1}$ & 18.30 & $17.66(18.96)$ & 1.00 & $17.65(18.86)$ & 20.83 & 1.00 & 20.82 \\
\hline \multirow[t]{15}{*}{ pyridine } & $1 \mathrm{a}_{2}$ & 9.60 & $8.34(9.60)$ & 1.00 & $8.33(9.60)$ & 9.41 & 1.00 & 9.40 \\
\hline & $11 \mathrm{a}_{1}$ & 9.75 & $8.75(10.01)$ & 1.00 & $8.91(10.18)$ & 11.13 & 0.99 & 11.37 \\
\hline & $2 b_{1}$ & 10.51 & $9.17(10.44)$ & 0.99 & $9.12(10.39)$ & 10.51 & 0.97 & 10.38 \\
\hline & $7 b_{2}$ & 12.61 & 11.79 (13.05) & 1.00 & $11.78(13.05)$ & 14.11 & 1.00 & 14.10 \\
\hline & $1 b_{1}$ & 13.10 & $12.53(13.79)$ & 1.00 & $12.54(13.81)$ & 14.62 & 1.00 & 14.62 \\
\hline & $10 \mathrm{a}_{1}$ & 13.80 & $13.06(14.33)$ & 1.00 & $13.05(14.32)$ & 15.62 & 1.00 & 15.65 \\
\hline & $6 b_{2}$ & 14.50 & $13.68(14.94)$ & 1.00 & $13.67(14.94)$ & 16.21 & 1.00 & 16.22 \\
\hline & $9 a_{1}$ & 15.90 & $14.98(16.25)$ & 1.00 & $14.96(16.23)$ & 17.78 & 1.00 & 17.76 \\
\hline & $5 b_{2}$ & 15.90 & $15.18(16.44)$ & 1.00 & $15.19(16.46)$ & 17.89 & 1.00 & 17.89 \\
\hline & $8 a_{1}$ & 17.40 & $16.64(17.90)$ & 1.00 & $16.62(17.89)$ & 19.54 & 1.00 & 19.55 \\
\hline & $4 b_{2}$ & 19.80 & $19.72(20.98)$ & 1.00 & $19.72(20.99)$ & 23.24 & 1.00 & 23.23 \\
\hline & $7 a_{1}$ & 20.60 & $19.74(21.00)$ & 1.00 & $19.72(20.99)$ & 23.31 & 1.00 & 23.30 \\
\hline & $3 b_{2}$ & 23.40 & $23.76(25.03)$ & 1.00 & $23.75(25.02)$ & 28.06 & 1.00 & 28.05 \\
\hline & $6 a_{1}$ & 24.50 & $25.10(26.36)$ & 1.00 & $25.08(26.35)$ & 29.61 & 1.00 & 29.58 \\
\hline & $5 a_{1}$ & 28.00 & $29.07(30.33)$ & 1.00 & $29.09(30.36)$ & 34.16 & 1.00 & 34.14 \\
\hline $\operatorname{MAD}^{\mathrm{d})}(\mathrm{eV})$ & & & $1.14(0.57)$ & & $1.15(0.54)$ & 1.76 & & 1.71 \\
\hline
\end{tabular}

a) Symmetry of the orbital undergoing ionization.

b) In parentheses, the ionization energy corrected for the difference between the calculated and the experimental first ionization energy, $I E_{k}^{\text {corr. }}=I E_{k}^{D F T}+\left(I E_{1}^{\text {exp. }}-I E_{1}^{D F T}\right)$.

c) Square norm $\left|\gamma_{k}\right|^{2}$ of the respective Dyson's orbital.

d) Mean absolute deviation of the computed ionization energies from the experimental values.

Table 1 collects the ground state EKT-SSR $\|$ vertical ionization energies of $\mathrm{HF}, \mathrm{HCl}$, $\mathrm{H}_{2} \mathrm{CO}, \mathrm{H}_{2} \mathrm{CS}, \mathrm{C}_{2} \mathrm{H}_{4}$, furan, thiophene, and pyridine. The experimental geometries were used as reported on the CCCBDB website. ${ }^{68}$ With the exception of the $\mathrm{HF}$ and $\mathrm{HCl}$ molecules, the active space in the SSR $(2,2)$ calculations comprises the HOMO and the LUMO orbitals obtained in the conventional single determinant RKS/RHF calculation with the same (BH\&HLYP or HF) functional. For $\mathrm{HF}$ and $\mathrm{HCl}$, the highest occupied $\sigma$-type molecular orbital and the lowest unoccupied $\sigma$-type molecular orbital were taken to the active

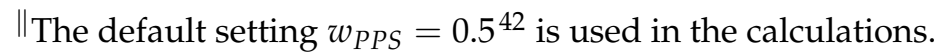


space. The EKT-SSR ionization energies are compared in Table 1 with the ionization energies obtained by the application of the Koopmans' theorem to the respective RKS/RHF computation, i.e., the negatives of the RKS/RHF orbital energies are used in lieu of the ionization energies; the latter will be denoted as the KT ionization energies, in the following.

For both energy functionals, the EKT-SSR and the KT ionization energies agree well with each other; typical deviations are less than $0.1 \mathrm{eV}$. In all cases, the norm of the Dyson's orbitals $\left|\gamma_{k}\right|^{2}$ is very close to unity. This implies that the ground electronic state of the neutral molecule is well represented by a single KS determinant and that there is a nearly perfect overlap between the ion orbitals and the corresponding orbitals of the neutral molecule. ${ }^{65}$ As was observed previously by Politzer and Abu-Awwad ${ }^{67}$ and Chong et al. ${ }^{65}$ the DFT valence ionization energies shifted to compensate for the difference between the calculated and the experimental first ionization energy, i.e., $I E_{k}^{c o r r}$. $=$ $I E_{k}^{D F T}+\left(I E_{1}^{\text {exp }}-I E_{1}^{D F T}\right)$ (given parenthetically in Table 1), match quite well the experimental values.** Although a proper way to ameliorate the deficiencies of the standard GGA and hybrid XC functionals is to reparameterize them such that (the negatives of) the KS orbital energies match the experimental ionization energies, ${ }^{69}$ the current approach (shifting) seems sufficient for the purpose of validation of the EKT-SSR ionization energies and demonstration of the capabilities of the method.

\subsection{Valence ionization energies of ozone}

Ozone $\mathrm{O}_{3}$ is a molecule with a mild diradical characteristic of its ground electronic state. According to the previous studies, ${ }^{61-63}$ the ground state of ozone can be sufficiently well

\footnotetext{
** In case when the experimental first ionization energies are not available, the suitable reference values can be obtained from a $\triangle \mathrm{SCF}$ calculation.
} 
approximated by a PPS configuration $C_{1}\left|\ldots\left(1 b_{1}\right)^{2}\left(1 a_{2}\right)^{2}\right\rangle-C_{2}\left|\ldots\left(1 b_{1}\right)^{2}\left(2 b_{1}\right)^{2}\right\rangle$. Recent ab initio multi-reference configurational interaction calculations ${ }^{63}$ predicted that the diradical characteristic of ozone ground state amounts to 0.18 ; the diradical characteristic was defined in Ref. 63 as $2 C_{2}^{2} /\left(C_{1}^{2}+C_{2}^{2}\right)$.

The vertical ionization energies of ozone were previously calculated using a variety of methods. ${ }^{61,70-75}$ The experimental assignment of the symmetries of the lowest ionization energies of ozone was given by Katsumata et al. ${ }^{76}$ using the angle resolved photoelectron spectroscopy (ARPES). Table 2 collects the experimentally measured valence vertical ionization energies of ozone in the gas phase and compares them with the energies obtained in the present work.

Table 2: Valence vertical ionization energies (eV) of ozone.

\begin{tabular}{|c|c|c|c|c|c|c|c|c|c|}
\hline \multicolumn{2}{|c|}{ Exp., Ref. 76} & \multicolumn{2}{|c|}{ RBH\&HLYP } & \multicolumn{3}{|c|}{ SSR-BH\&HLYP $(0.50)^{b)}$} & \multicolumn{3}{|c|}{ SSR-BH\&HLYP(0.99) } \\
\hline Symm. ${ }^{\text {a) }}$ & IE & Symm. & IE & Symm. & $\mathrm{IE}^{\mathrm{C})}$ & norm $^{\mathrm{d})}$ & Symm. & IE & norm \\
\hline $6 a_{1}$ & 12.73 & $1 a_{2}$ & 11.41 (12.73) & $6 a_{1}$ & 11.67 (12.73) & 1.00 & $6 a_{1}$ & 11.61 (12.73) & 1.00 \\
\hline $4 b_{2}$ & 13.00 & $6 a_{1}$ & $11.77(13.10)$ & $1 \mathrm{a}_{2}$ & $12.00(13.05)$ & 0.83 & $4 b_{2}$ & $12.10(13.22)$ & 1.00 \\
\hline $1 a_{2}$ & 13.54 & $4 b_{2}$ & $11.99(13.31)$ & $4 b_{2}$ & $12.23(13.29)$ & 1.00 & $1 \mathrm{a}_{2}$ & $12.84(13.96)$ & 0.81 \\
\hline $2 b_{1}$ & 16.50 & & & $2 b_{1}$ & $11.49(12.55)$ & 0.33 & $2 b_{1}$ & $12.86(13.98)$ & 0.36 \\
\hline
\end{tabular}

a) Symmetry of the orbital undergoing ionization.

b) In parentheses, the value of the $w_{P P S}$ weighting factor in the SA-REKS functional, Eq. (3).

c) In parentheses, the ionization energy corrected for the difference between the calculated and the experimental first ionization energy, $I E_{k}^{\text {corr. }}=I E_{k}^{D F T}+\left(I E_{1}^{\text {exp. }}-I E_{1}^{D F T}\right)$.

d) Square norm $\left|\gamma_{k}\right|^{2}$ of the respective Dyson's orbital.

The SSR(2,2)-BH\&HLYP/6-311G** calculation employing the experimental geometry of ozone ${ }^{68}$ and taking the $1 a_{2}$ and $2 b_{1}$ orbitals into the active space agrees with the previous multi-reference calculations that ozone has a mild diradical characteristic. The occu- 
pation numbers of the $1 a_{2}$ and $2 b_{1}$ natural orbitals obtained from the ground state relaxed density matrix of ozone are 1.66 and 0.31 , respectively; this lends ozone an 0.31 diradical characteristic.

The calculated valence vertical ionization energies of ozone are reported in Table 2 along with their respective pole strengths (square norms of the Dyson's orbitals). With the use of the default settings of the $\operatorname{SSR}(2,2)$ method, which employs the ensemble weighting factor $w_{P P S}=0.5$, the Dyson's orbitals with strong ionization probability are ordered as $6 a_{1}<1 a_{2}<4 b_{2}$. This ordering of the ionization states is an improvement over the KT ordering $1 a_{2}<6 a_{1}<4 b_{2}$, however contradicts the experimental ordering $6 a_{1}<4 b_{2}<$ $1 a_{2}$.

The origin of the discrepancy may be traced back to the dependence of the energies of the EKT-SSR Dyson's orbitals on the choice of the ensemble weighting factor $w_{P P S}$ in Eq. (3). Indeed, the default choice $w_{P P S}=0.5$ of the ensemble weighting factor is better suited for describing the excited states, rather than the ground states of molecules. ${ }^{42,49}$ Shifting $w_{P P S}$ to favor the ground electronic state in the ensemble, e.g., $w_{P P S}=0.99$, improves the description of the ground state properties. In particular, the ordering of the $1 a_{2}$ and $4 b_{2}$ Dyson's orbitals is reversed and agrees with the experiment.

The $1 a_{2}$ Dyson's orbital has a reduced pole strength $(0.81)$, which is not unexpected in view of the discussion at the beginning of the Section 3. Indeed, the ground state density of ozone has fractionally occupied $1 a_{2}$ and $2 b_{1}$ natural orbitals and this should lead to fractional norms of the respective Dyson's orbitals. Interestingly, the $2 b_{1}$ Dyson's orbital (with $w_{P P S}=0.99$ ) occurs below the outer valence Dyson's orbitals. This Dyson's orbital has a small norm (0.36) and corresponds to a shake-up ionization state, where the electron removal from the $1 a_{2}$ orbital is accompanied by promotion of the remaining electron to 
the $2 b_{1}$ orbital.

The occurrence of a $2 b_{1}$ satellite ionization state is confirmed by the experimental measurement, ${ }^{76}$ which predicts its ionization energy at $c a .16 .50 \mathrm{eV}$, and by the high level multi-reference computations, ${ }^{75}$ which put a $b_{1}$-symmetric state with very low ionization probability $(0.139)$ at $16.75 \mathrm{eV}$. Although the quantitative agreement of the EKTSSR/BH\&HLYP/6-311G** $2 b_{1}$ energy $(13.98 \mathrm{eV})$ with the experimental and theoretical values seems quite poor, the exact positioning of the $2 b_{1}$ Dyson's orbital may depend on the density functional employed. The investigation of the dependence of the EKT-SSR ionization energies on the choice of the density functional is however beyond the scope of the present work.

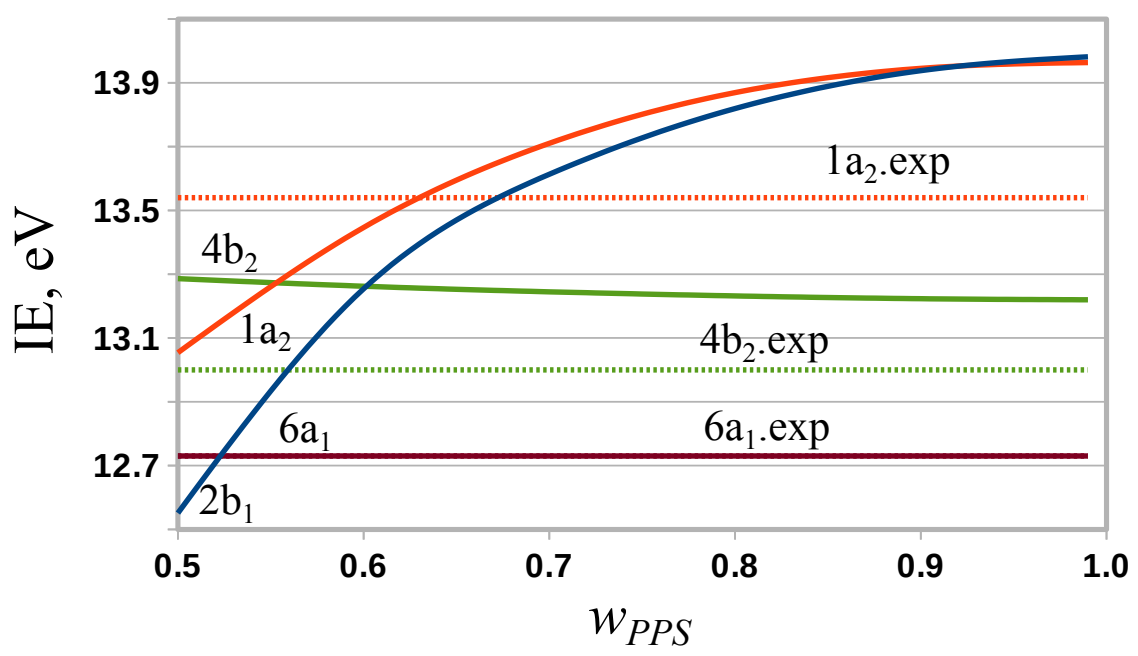

Figure 1: Valence vertical ionization energies (in $\mathrm{eV}$ ) of ozone obtained using the SSRBH\&HLYP $/ 6-311 \mathrm{G}^{* *}$ method with varying $w_{P P S}$ ensemble weighting factor. The ionization energies are shifted to match the experimental first ionization energy; see caption to Tables 1 and 2.

The energies of the $2 b_{1}$ and $1 a_{2}$ Dyson's orbitals display a pronounced dependence on the choice of the state-averaging weighting factor $w_{P P S}$, see Fig. 1 . In the state-averaged 
methods, such as the SA-CASSCF method, the individual state energies and properties are typically dependent on the ensemble weighting factors. ${ }^{53}$ As suggested by Stålring et al., ${ }^{53}$ the energies of the SA individual states can be corrected by adding the first-order derivatives of the SA energies on the weighting factors to obtain better estimates for the individual states. In the parlance of eDFT this would correspond to the inclusion of the dependence on the ensemble weighting factors into the XC density functionals. ${ }^{33}$ The weight dependence of the ensemble XC functionals is an ongoing field of research ${ }^{33,34}$ and the results in Fig. 1 underline the need for development of XC functionals designed for eDFT calculations. As the standard (weight-independent) XC functionals, such as BH\&HLYP, were designed for the ground state computations only, better estimates of the ground state properties of multi-reference systems can be obtained with the choice of the ensemble weighting factor $w_{P P S}$ favoring the ground electronic state. As for the molecules reported in Table 1, which have single-reference closed-shell ground electronic states, the choice of $w_{P P S}$ is less significant; increasing $w_{P P S}$ to 0.95 affects the reported ionization energies by less than $0.1 \mathrm{eV}$. Hence, the default choice $w_{P P S}=0.5$ (an equi-ensemble of the ground and excited states) is suitable for the rest of the computations presented in this work.

\subsection{Vertical ionization energies of the excited states}

An important advantage of the EKT-SSR methodology is that it allows to calculate the ionization energies of the excited state, e.g., during propagation of the non-adiabatic molecular dynamics (NAMD) trajectories. Indeed, all the quantities needed in Eq. (15) are present in Eq. (11), which provides the gradient of the target state. Hence, the ionization energies of the excited state can be obtained at no additional cost. Although we did not 
manage to find any experimental reference data for the ionization energies of the excited states, it seems useful to report these energies anyway. Table 3 collects the ionization energies of the excited states of the molecules reported in Table 1; the target excited state is obtained by the $\mathrm{HOMO} \rightarrow$ LUMO transition and is included in the state-averaging in Eq. (3).

Table 3: Vertical ionization energies (in $\mathrm{eV}$ ) of singlet excited states of the molecules reported in Table 1 calculated using the SSR-BH\&HLYP/6-311G** and SSR-HF/6-311G** methods. Theoretical best estimates (TBE, in $\mathrm{eV}$ ) of the vertical excitation energies (VEE) are taken from Refs. 77 and 78. In parentheses are given the DFT ionization energies corrected for the difference between the calculated and the experimental first ionization energies of the ground state. See legend to Tables 1 and 2 for detail.

\begin{tabular}{|c|c|c|c|c|c|c|c|c|c|c|}
\hline \multirow{2}{*}{ Molecule } & \multirow{2}{*}{ transition } & \multirow{2}{*}{$\mathrm{TBE}$} & \multicolumn{4}{|c|}{ EKT-SSR-BH\&HLYP } & \multicolumn{4}{|c|}{ EKT-SSR-HF } \\
\hline & & & VEE & Symm. & IE & norm & VEE & Symm. & IE & norm \\
\hline \multirow[t]{6}{*}{$\mathrm{H}_{2} \mathrm{CO}$} & $n \rightarrow \pi^{*}$ & 3.97 & 4.00 & $2 b_{1}$ & $5.08(6.73)$ & 0.50 & 3.71 & $2 b_{1}$ & 7.16 & 0.49 \\
\hline & & & & $2 b_{2}$ & $10.63(12.29)$ & 0.51 & & $2 b_{2}$ & 13.39 & 0.58 \\
\hline & & & & $1 b_{1}$ & $13.54(15.19)$ & 1.00 & & $1 b_{1}$ & 16.76 & 1.01 \\
\hline & & & & $5 a_{1}$ & $15.25(16.90)$ & 1.00 & & $1 b_{2}$ & 18.15 & 0.92 \\
\hline & & & & $1 b_{2}$ & $15.41(17.06)$ & 0.99 & & $5 a_{1}$ & 18.37 & 1.00 \\
\hline & & & & $4 a_{1}$ & $19.59(21.24)$ & 1.00 & & $4 a_{1}$ & 23.24 & 1.00 \\
\hline \multirow[t]{6}{*}{$\mathrm{H}_{2} \mathrm{CS}$} & $n \rightarrow \pi^{*}$ & 2.20 & 2.27 & $3 b_{1}$ & $5.38(7.01)$ & 0.49 & 1.99 & $3 b_{1}$ & 7.13 & 0.50 \\
\hline & & & & $3 b_{2}$ & $8.97(10.59)$ & 0.50 & & $3 b_{2}$ & 10.81 & 0.50 \\
\hline & & & & $2 b_{1}$ & $10.25(11.87)$ & 1.00 & & $2 b_{1}$ & 12.11 & 0.99 \\
\hline & & & & $7 a_{1}$ & $12.62(14.24)$ & 1.00 & & $7 a_{1}$ & 14.75 & 1.00 \\
\hline & & & & $2 b_{2}$ & $14.14(15.76)$ & 0.99 & & $2 b_{2}$ & 16.74 & 0.99 \\
\hline & & & & $6 a_{1}$ & $18.49(20.11)$ & 1.00 & & $6 a_{1}$ & 21.74 & 1.00 \\
\hline \multirow[t]{7}{*}{$\mathrm{C}_{2} \mathrm{H}_{4}$} & $\pi \rightarrow \pi^{*}$ & 7.92 & 7.93 & $1 b_{2 g}$ & $0.97(3.02)$ & 0.50 & 9.27 & $1 b_{2 g}$ & 0.37 & 0.50 \\
\hline & & & & $1 b_{3 u}$ & $7.80(9.85)$ & 0.50 & & $1 b_{3 u}$ & 8.66 & 0.51 \\
\hline & & & & $1 b_{3 g}$ & $11.81(13.86)$ & 1.00 & & $1 b_{3 g}$ & 14.25 & 1.00 \\
\hline & & & & $3 a_{g}$ & $13.90(15.94)$ & 1.00 & & $3 a_{g}$ & 16.62 & 1.00 \\
\hline & & & & $1 b_{2 u}^{\circ}$ & 15.29 (17.34) & 1.00 & & $1 b_{2 u}^{\circ}$ & 18.11 & 1.00 \\
\hline & & & & $2 b_{1 u}$ & $18.48(20.53)$ & 1.00 & & $2 b_{1 u}$ & 22.01 & 1.00 \\
\hline & & & & $2 a_{g}$ & $24.40(26.45)$ & 1.00 & & $2 a_{g}$ & 28.79 & 1.00 \\
\hline \multirow[t]{12}{*}{ furan } & $\pi \rightarrow \pi^{*}$ & 6.37 & 6.47 & $3 b_{1}$ & $1.03(2.65)$ & 0.51 & 7.40 & $3 b_{1}$ & 0.93 & 0.52 \\
\hline & & & & $1 a_{2}$ & $6.95(8.57)$ & 0.50 & & $1 a_{2}$ & 8.04 & 0.50 \\
\hline & & & & $2 b_{1}$ & $9.23(10.85)$ & 0.99 & & $2 b_{1}$ & 11.15 & 0.99 \\
\hline & & & & $9 a_{1}$ & $12.10(13.72)$ & 1.00 & & $9 a_{1}$ & 14.68 & 1.00 \\
\hline & & & & $8 a_{1}$ & $12.81(14.43)$ & 1.00 & & $8 a_{1}$ & 15.43 & 1.00 \\
\hline & & & & $6 b_{2}$ & $13.48(15.10)$ & 1.00 & & $6 b_{2}$ & 16.26 & 1.00 \\
\hline & & & & $5 b_{2}$ & 14.13(15.75) & 1.00 & & $5 b_{2}$ & 16.65 & 1.00 \\
\hline & & & & $1 b_{1}$ & 14.17(15.79) & 0.99 & & $1 b_{1}$ & 16.97 & 0.98 \\
\hline & & & & $7 a_{1}$ & $17.15(18.76)$ & 1.00 & & $7 a_{1}$ & 20.28 & 1.00 \\
\hline & & & & $6 a_{1}$ & 18.14(19.76) & 1.00 & & $6 a_{1}$ & 21.66 & 1.00 \\
\hline & & & & $4 b_{2}$ & 18.81(20.42) & 1.00 & & $4 b_{2}$ & 22.11 & 1.00 \\
\hline & & & & $3 b_{2}$ & $23.36(24.98)$ & 1.00 & & $3 b_{2}$ & 27.63 & 1.00 \\
\hline
\end{tabular}


Table 3 - Continued from previous page

\begin{tabular}{|c|c|c|c|c|c|c|c|c|c|c|}
\hline \multirow{2}{*}{ Molecule } & \multirow{2}{*}{ transition } & \multirow{2}{*}{ TBE } & \multicolumn{4}{|c|}{ EKT-SSR-BH\&HLYP } & \multicolumn{4}{|c|}{ EKT-SSR-HF } \\
\hline & & & VEE & Symm. & IE & norm & VEE & Symm. & IE & norm \\
\hline \multirow[t]{11}{*}{ thiophene } & $\pi \rightarrow \pi^{*}$ & 5.63 & 6.13 & $4 b_{1}$ & $1.55(2.84)$ & 0.51 & 7.12 & $4 b_{1}$ & 1.45 & 0.52 \\
\hline & & & & $1 a_{2}$ & $7.18(8.47)$ & 0.50 & & $1 a_{2}$ & 8.20 & 0.50 \\
\hline & & & & $3 b_{1}$ & $8.17(9.46)$ & 0.99 & & $3 b_{1}$ & 9.59 & 0.99 \\
\hline & & & & $11 a_{1}$ & 10.90(12.19) & 1.00 & & $11 a_{1}$ & 12.91 & 1.00 \\
\hline & & & & $2 b_{1}$ & $12.03(13.33)$ & 0.99 & & $2 b_{1}$ & 14.04 & 0.98 \\
\hline & & & & $7 b_{2}$ & $12.40(13.69)$ & 1.00 & & $7 b_{2}$ & 14.54 & 1.00 \\
\hline & & & & $10 \bar{a}_{1}$ & $12.75(14.04)$ & 1.00 & & $10 \bar{a}_{1}$ & 15.19 & 1.00 \\
\hline & & & & $6 b_{2}$ & 13.47(14.77) & 1.00 & & $6 b_{2}$ & 16.03 & 1.00 \\
\hline & & & & $9 a_{1}$ & $16.31(17.60)$ & 1.00 & & $9 a_{1}$ & 19.17 & 1.00 \\
\hline & & & & $5 b_{2}$ & $17.52(18.81)$ & 1.00 & & $5 b_{2}$ & 20.64 & 1.00 \\
\hline & & & & $8 a_{1}$ & 17.87(19.17) & 1.00 & & $8 a_{1}$ & 21.11 & 1.00 \\
\hline \multirow[t]{16}{*}{ pyridine } & $n \rightarrow \pi^{*}$ & 4.95 & 5.27 & $3 b_{1}$ & $3.50(4.76)$ & 0.50 & 5.40 & $3 b_{1}$ & 4.41 & 0.49 \\
\hline & & & & $1 a_{2}$ & $8.41(9.67)$ & 1.00 & & $1 a_{2}$ & 9.66 & 1.00 \\
\hline & & & & $2 b_{1}$ & 9.30(10.57) & 1.00 & & $2 b_{1}$ & 10.94 & 1.00 \\
\hline & & & & $11 a_{1}$ & 10.31(11.58) & 0.53 & & $11 a_{1}$ & 12.99 & 0.64 \\
\hline & & & & $7 b_{2}$ & 11.71(12.97) & 1.00 & & $7 b_{2}$ & 13.98 & 1.00 \\
\hline & & & & $10 a_{1}$ & $12.66(13.92)$ & 0.97 & & $10 a_{1}$ & 15.26 & 0.88 \\
\hline & & & & $1 b_{1}$ & 13.09(14.35) & 1.00 & & $1 b_{1}$ & 15.67 & 1.00 \\
\hline & & & & $6 b_{2}$ & 13.63(14.90) & 1.00 & & $6 b_{2}$ & 16.15 & 1.00 \\
\hline & & & & $9 a_{1}$ & $14.89(16.15)$ & 1.00 & & $9 a_{1}$ & 17.64 & 0.99 \\
\hline & & & & $5 b_{2}$ & $15.19(16.45)$ & 1.00 & & $5 b_{2}$ & 17.87 & 1.00 \\
\hline & & & & $8 a_{1}$ & $16.50(17.77)$ & 0.99 & & $8 a_{1}$ & 19.32 & 0.98 \\
\hline & & & & $7 a_{1}$ & $19.46(20.72)$ & 1.00 & & $7 a_{1}$ & 22.98 & 0.99 \\
\hline & & & & $4 b_{2}$ & $19.92(21.18)$ & 1.00 & & $4 b_{2}$ & 23.43 & 1.00 \\
\hline & & & & $3 b_{2}$ & $23.81(25.07)$ & 1.00 & & $3 b_{2}$ & 28.08 & 1.00 \\
\hline & & & & $6 a_{1}$ & $24.77(26.03)$ & 1.00 & & $6 a_{1}$ & 29.21 & 0.99 \\
\hline & & & & $5 a_{1}$ & 29.72(30.98) & 1.00 & & $5 a_{1}$ & 34.79 & 1.00 \\
\hline
\end{tabular}

The vertical excitation energies calculated with the SSR-BH\&HLYP /6-311G** method are in a good agreement with the most recent theoretical best estimate (TBE) values. ${ }^{77,78}$ The DFT ionization energies in Table 3 are corrected for the difference between the experimental and calculated first ionization potential of the ground state, i.e., $I E_{k}^{\text {corr. }}\left(S_{1}\right)=$ $I E_{k}^{D F T}\left(S_{1}\right)+\left(I E_{1}^{\text {exp. }}\left(S_{0}\right)-I E_{1}^{D F T}\left(S_{0}\right)\right)$; the corrected values are given parenthetically. Such a procedure yields very close values for the inner valence ionization energies of the $S_{0}$ and $S_{1}$ states of the molecules in Tables 1 and 3 . The outer valence ionization energies in the $S_{1}$ state are split into two ionization states with reduced intensity (the squared norm of the respective Dyson's orbitals). According to the arguments at the beginning of the Section 3, this should be expected for an OSS-type excited state. As the targeted excited states are well approximated by an OSS-type configuration, see Eq. (19), two ionization channels 
are possible, when removing electrons from the fractionally occupied KS orbitals: In the first channel, the resulting ion has the same electronic configuration as in the case of the $\mathrm{S}_{0}$ ionization, whereas in the second channel the electron in the ion is promoted to the KS orbital matching the LUMO of the $\mathrm{S}_{0}$ state.

All the ionization channels in the outer and inner valence region can be calculated using the EKT-SSR method. As in the time-resolved PES (TRPES) experiments a short XUV or X-ray pulses are used for the ionization, the knowledge of multiple possible ionization channels may be required for modeling the ionization cross-sections theoretically.

\subsection{Ionization energies during bond dissociation: $\mathrm{H}_{2}$ and $\mathrm{LiH}$}

During homolytic dissociation of a covalent bond a gradual transition from a single reference to a multi-reference description of the ground electronic state occurs as the bond is stretched. In the textbook example of $\mathrm{H}_{2}$, the ground ${ }^{1} \Sigma_{g}^{+}$electronic state is well approximated by a PPS-type configuration $C_{1}\left|1 \sigma_{g} \overline{1 \sigma}_{g}\right\rangle-C_{2}\left|1 \sigma_{u} \overline{1 \sigma}_{u}\right\rangle$, where $1 \sigma_{g}$ and $1 \sigma_{u}$ are the bonding and the anti-bonding $\sigma$-type orbitals. Near the equilibrium bondlength, the $C_{1}$ coefficient is dominant and the electronic structure is well approximated by a single determinant $\left|1 \sigma_{g} \overline{1}_{g}\right\rangle$. As the bond is stretched, the $\left|1 \sigma_{u} \overline{1 \sigma}_{u}\right\rangle$ configuration gains more weight and, in the limit of the fully dissociated bond, $C_{2}=C_{1}=1 / \sqrt{2}$. As the ground state electronic configuration now corresponds to two neutral atoms in their ground electronic states, the energies of the Dyson's orbitals should converge to their atomic values. The excited ${ }^{1} \Sigma_{u}^{+}$electronic state of $\mathrm{H}_{2}$ is well approximated by an OSS-type configuration $1 / \sqrt{2}\left|1 \sigma_{g} \overline{1 \sigma}_{u}\right\rangle+1 / \sqrt{2}\left|1 \sigma_{u} \overline{1 \sigma}_{g}\right\rangle$ and, in the dissociation limit, it corresponds to a superposition of two ionic Lewis structures $\mathrm{H}^{+}-\mathrm{H}^{-}$and $\mathrm{H}^{-}-\mathrm{H}^{+}$. Hence, the energy of the Dyson's orbitals is expected to converge to the orbital energy of the negative ion $\mathrm{H}^{-}$. 

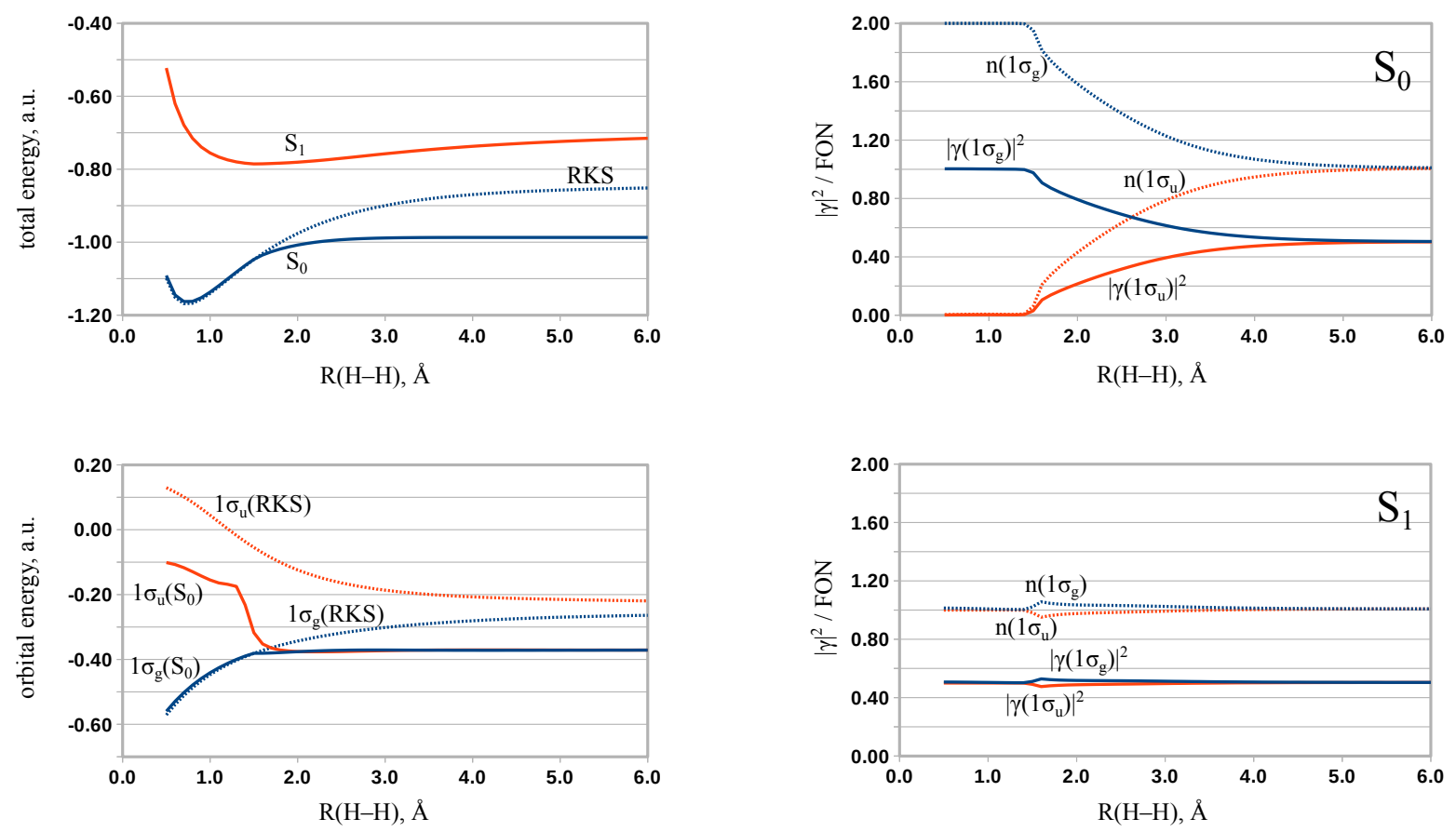

Figure 2: Dissociation of the $\mathrm{H}_{2}$ molecule. Upper left panel: The total energies of the $\mathrm{S}_{0}$ and $S_{1}$ states obtained with SSR-BH\&HLYP/6-311G ${ }^{* *}$ (solid lines) and the total energy of the $S_{0}$ state from RBH\&HLYP/6-311G ${ }^{* *}$ (dotted line) as function of the internuclear distance. Lower left panel: The energies of the $1 \sigma_{g}$ and $1 \sigma_{u}$ Dyson's orbitals in the $S_{0}$ state from the EKT-SSR calculation (solid lines) and from the KT calculation (dotted line). Upper right panel: the FONs of the active orbitals in the SSR-BH\&HLYP/6-311G ${ }^{* *}$ calculation and the norms of the respective Dyson's orbitals in the $\mathrm{S}_{0}$ state. Lower right panel: The same for the $S_{1}$ state.

Fig. 2 shows dissociation curves of the ${ }^{1} \Sigma_{g}^{+}\left(\mathrm{S}_{0}\right)$ and the ${ }^{1} \Sigma_{u}^{+}\left(\mathrm{S}_{1}\right)$ states of $\mathrm{H}_{2}$ calculated using the SSR-BH\&HLYP/6-311G** method. As is well known, the standard single reference RKS method is not able to correctly describe the dissociation of the $\mathrm{H}_{2}$ molecule, as well as the standard linear response TD-DFT is not capable of describing the potential energy curve (PEC) of the $S_{1}$ state correctly; ${ }^{17,18,42,79}$ see the dotted curve in the upper left panel of Fig. 2. In the lower left panel of Fig. 2 the energies of the $1 \sigma_{g}$ and the $1 \sigma_{u}$ orbitals are shown as obtained by the EKT-SSR-BH\&HLYP $/ 6-311 \mathrm{G}^{* *}$ method. In the limit of the 
dissociated $\mathrm{H}-\mathrm{H}$ bond, the energies of the Dyson's orbitals (solid curves) converge to the same value, which corresponds to the orbital energy of an isolated $\mathrm{H}$ atom obtained, e.g., in a spin-unrestricted BH\&HLYP calculation. The orbital energies from an RKS calculation converge to a higher orbital energy in the limit of a dissociated bond.

The rightmost panels of Fig. 2 show the dependence of the norms $|\gamma|^{2}$ of the $1 \sigma_{g}$ and the $1 \sigma_{u}$ Dyson's orbitals on the $\mathrm{H}-\mathrm{H}$ bond length. In the $\mathrm{S}_{0}$ state, the norms remain constant at the values of 0 and 1 until the Coulson-Fischer point ${ }^{80}$ is reached at $c a .1 .5$ $\AA$. Then, the norm of the $1 \sigma_{g}$ Dyson's orbital departs from unity and gradually decreases to the value of 0.5 ; simultaneously the norm of the $1 \sigma_{u}$ orbital begins to increase and reaches the value of 0.5 in the limit of a dissociated bond. As discussed in the beginning of the Section 3, this behavior is typical for a PPS-type configuration, where the weighting factors become equal in their magnitude.

The ${ }^{1} \Sigma_{u}^{+}$excited state of $\mathrm{H}_{2}$ is well approximated by an OSS-type configuration obtained by promoting one electron from the $1 \sigma_{g}$ to $1 \sigma_{u}$ orbital. In this case, the norms of the respective Dyson's orbitals remain (nearly) constant around a value of 0.5 , see the lower right panel of Fig. 2. It is noteworthy that the magnitudes of the norms of the Dyson's orbitals vary parallel with the (relaxed) FONs of the respective KS orbitals; the latter are shown by the dotted curves in the rightmost panels of Fig. 2. This observation agrees with the discussion at the beginning of the Section 3.

A more detailed view of the variation of the energies and norms of the $1 \sigma_{g}$ and $1 \sigma_{u}$ Dyson's orbitals of $\mathrm{H}_{2}$ is shown in Fig. 3. In this figure, the EKT-SSR-BH\&HLYP / 6-311G** energies of the Dyson's orbitals are shifted by the difference of the calculated (13.37 eV) and the experimental $(15.43 \mathrm{eV})$ first ionization energies of $\mathrm{H}_{2}$ at the $\mathrm{S}_{0}$ equilibrium distance. For both $S_{0}$ and $S_{1}$ states, the energies of the Dyson's orbitals converge to the same 

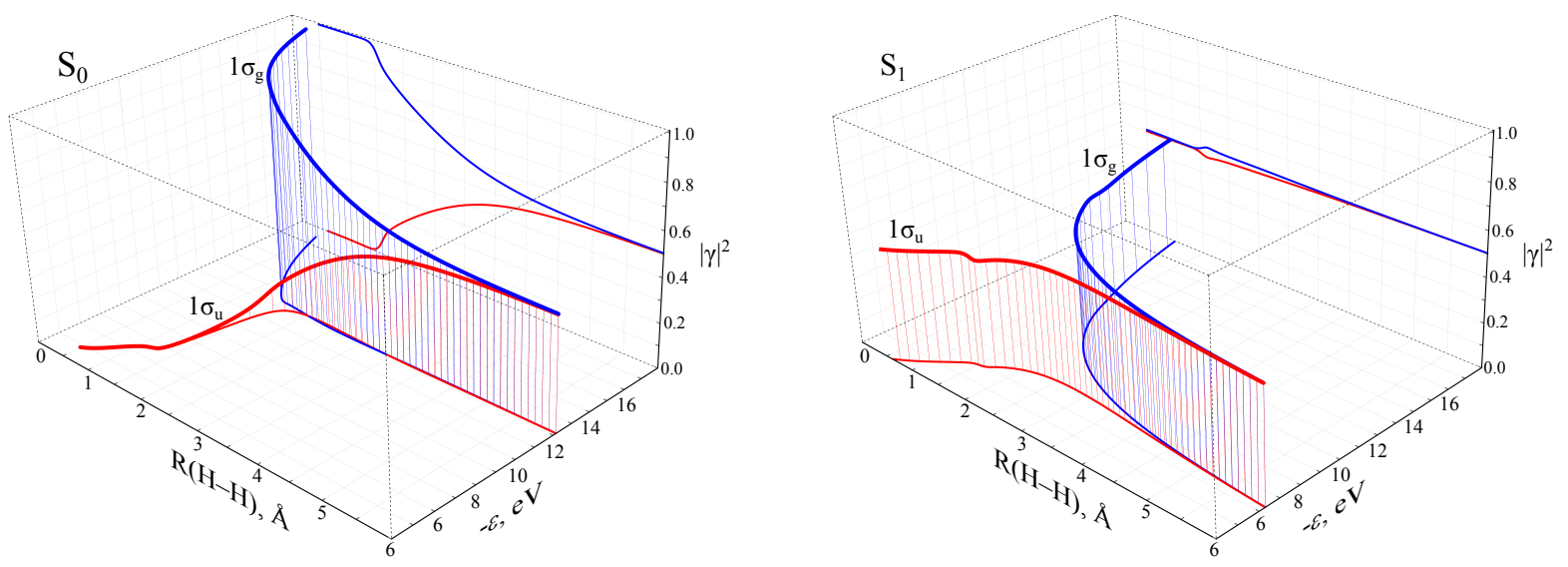

Figure 3: 3D plots showing the dependence of the energies and the norms of the $1 \sigma_{g}$ and $1 \sigma_{u}$ Dyson's orbitals in the $S_{0}$ state (left panel) and in the $S_{1}$ state (right panel) on the internuclear distance in $\mathrm{H}_{2}$.

value in the dissociation limit, as should be expected for the homolytic dissociation of a covalent bond.

A more interesting situation is observed in the dissociation of the $\mathrm{LiH}$ molecule, see Fig. 4. Near its ground state equilibrium geometry, the $\mathrm{S}_{0}$ state of LiH displays a mildly ionic characteristic (the Mulliken charge on the Li atom is +0.33 , as obtained from the relaxed SSR-BH\&HLYP/6-311G** density matrix), whereas the $S_{1}$ state has a covalent characteristic $\left(Q_{L i}=-0.06\right)$. At the $\mathrm{Li}-\mathrm{H}$ distance of $6 \AA, Q_{L i}$ in the $\mathrm{S}_{0}$ state decreases to +0.12 , whereas in the $S_{1}$ state it increases to +0.77 . This indicates that the $S_{0}$ and $S_{1}$ states swap the ionic and covalent characteristics; the $S_{0}$ state becomes covalent and the $\mathrm{S}_{1}$ state becomes ionic. Hence, at an intermediate distance of $c a$. 3.0-4.0 $\AA$, the two states undergo an avoided crossing, which is seen in the upper right panel of Fig. 4 where the SSR-BH\&HLYP/6-311G** PECs of the $S_{0}$ and $S_{1}$ states are shown.

The energies of the $2 \sigma$ (the $\sigma$-type bonding orbital) and the $3 \sigma$ (the anti-bonding orbital) Dyson's orbitals in the $S_{0}$ state also undergo an avoided crossing near $3.0 \AA$, see the lower left panel of Fig. 4. In the limit of dissociated $\mathrm{Li}-\mathrm{H}$ bond, the $S_{0}$ energies of these 

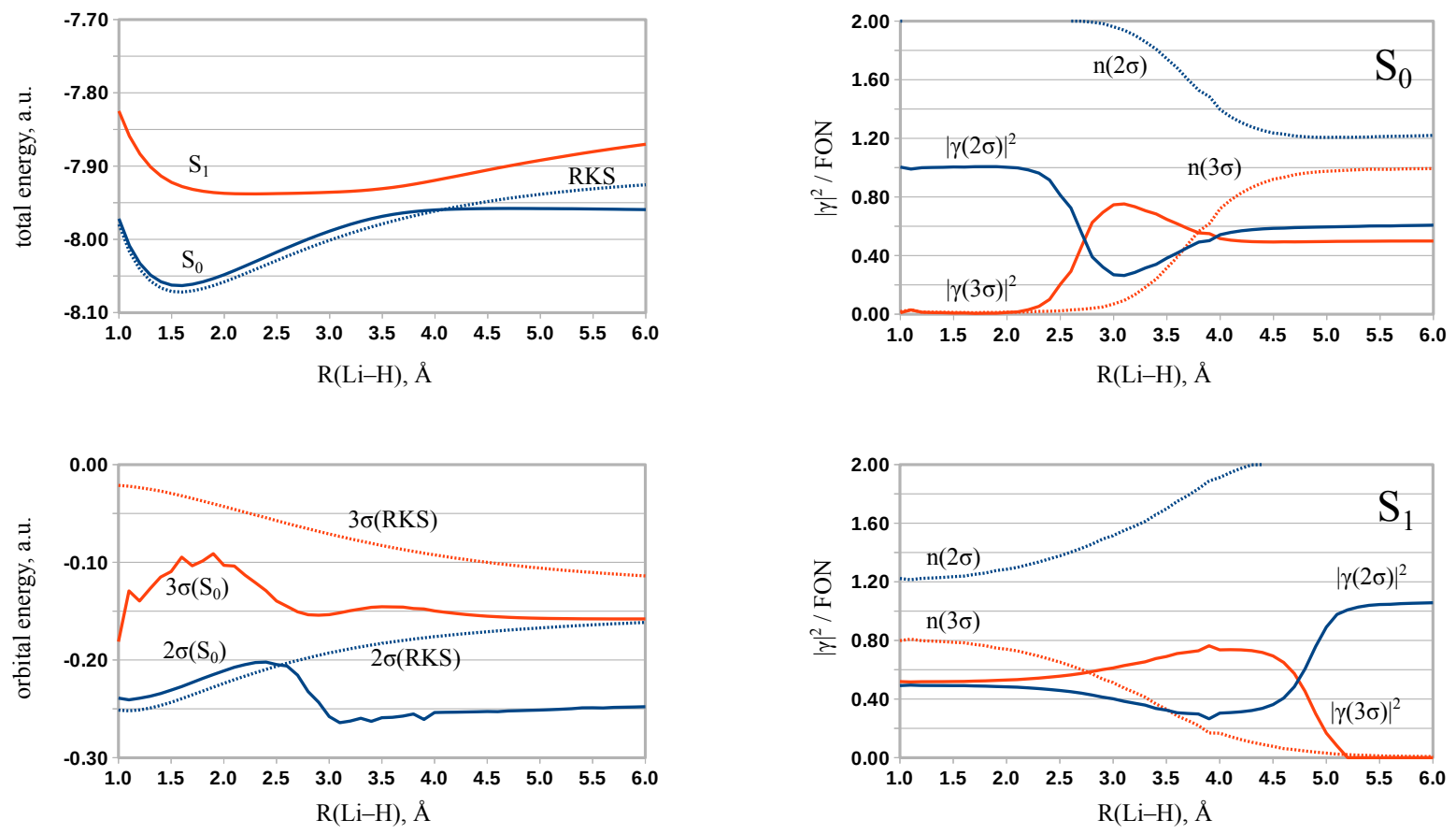

Figure 4: Dissociation of the LiH molecule. Upper left panel: The total energies of the $\mathrm{S}_{0}$ and $S_{1}$ states obtained with SSR-BH\&HLYP $/ 6-311 G^{* *}$ (solid lines) and the total energy of the $S_{0}$ state from RBH\&HLYP / 6-311G $\mathrm{G}^{* *}$ (dotted line) as function of the internuclear distance. Lower left panel: The energies of the $2 \sigma$ and $3 \sigma$ Dyson's orbitals in the $\mathrm{S}_{0}$ state from the EKT-SSR calculation (solid lines) and from the KT calculation (dotted line). Upper right panel: the FONs of the active orbitals in the SSR-BH\&HLYP/6-311G** calculation and the norms of the respective Dyson's orbitals in the $S_{0}$ state. Lower right panel: The same for the $S_{1}$ state.

orbitals converge to the frontier orbital energies of the neutral $\mathrm{Li}(3 \sigma)$ and $\mathrm{H}(2 \sigma)$ atoms. $^{\dagger \dagger}$ The usual single-reference RKS method does not reproduce the correct dissociation behavior of the $\mathrm{Li}-\mathrm{H}$ bond, nor does it reproduce the trends in the energies of the frontier

\footnotetext{
${ }^{\dagger \dagger}$ Note that, in Fig. 4, the energies of the Dyson's orbitals obtained with the default setting of the EKTSSR method with $w_{P P S}=0.5$ are shown. At $6.0 \AA$ these energies are $-0.2479(2 \sigma)$ and $-0.1580(3 \sigma)$ a.u.; the energies of the singly occupied orbitals of the neutral $\mathrm{H}$ and Li atoms obtained with U-BH\&HLYP/6$311 G^{* *}$ method are -0.3844 and -0.1559 a.u., respectively. Setting the ensemble weighting factor $w_{P P S}$ in the EKT-SSR-BH\&HLYP / 6-311G $\mathrm{G}^{* *}$ calculation to a value of 0.95 (i.e., single state instead of state averaged calculation) brings the energies of the Dyson's orbitals, $-0.3988(2 \sigma)$ and $-0.1576(3 \sigma)$ a.u., in close agreement with the atomic orbital energies.
} 
orbitals of $\mathrm{LiH}$; see the dotted curves in the leftmost panels of Fig. 4.
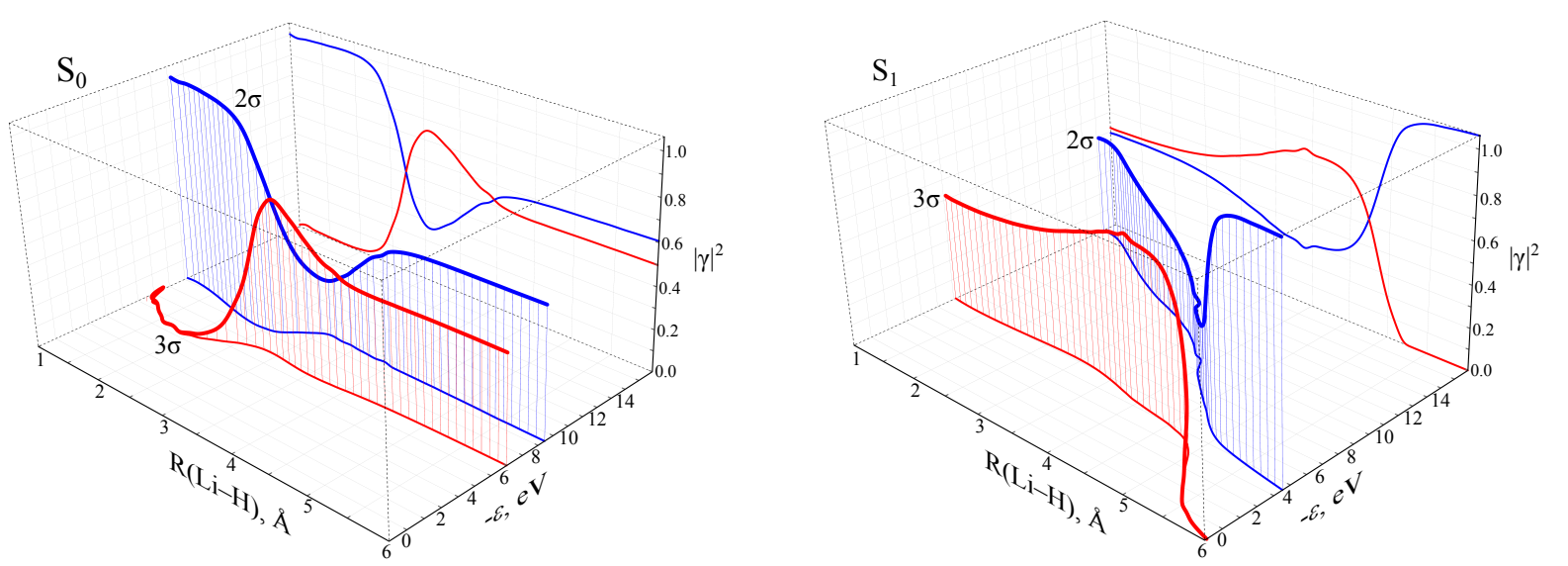

Figure 5: 3D plots showing the dependence of the energies and the norms of the $2 \sigma$ and $3 \sigma$ Dyson's orbitals in the $S_{0}$ state (left panel) and in the $S_{1}$ state (right panel) on the internuclear distance in $\mathrm{LiH}$.

Inspection of the norms $|\gamma|^{2}$ of Dyson's orbitals of LiH suggests that, at the onset of the $S_{1} / S_{0}$ avoided crossing at ca. 2.5-3.0 $\AA$, the formerly ionic $S_{0}$ state first becomes (nearly) covalent, $|\gamma(3 \sigma)|^{2} \approx|\gamma(2 \sigma)|^{2} \approx 0.5$, and then becomes slightly more ionic again, $|\gamma(3 \sigma)|^{2}>|\gamma(2 \sigma)|^{2}$, before becoming covalent in the dissociation limit, see the upper right panel in Fig. 4. A similar rise and fall of the Li-H bond ionicity is seen in the $\mathrm{S}_{1}$ state, only in the inverse order; the covalent state becomes slightly ionic before acquiring full ionicity in the limit of dissociated $\mathrm{Li}-\mathrm{H}$ bond. Note, that the $\mathrm{S}_{1}$ state of $\mathrm{LiH}$ in the dissociation limit corresponds to the $\mathrm{Li}^{+}-\mathrm{H}^{-}$Lewis structure; as this electronic structure is described by a single determinant electronic configuration, the norms of the Dyson's orbitals converge to 0 and 1, see the lower right panel in Fig. 4. The 3D plots in Fig. 5 show the behavior of both the Dyson's orbital energies ${ }^{\ddagger \ddagger}$ and their norms in the $S_{0}$ and the $\mathrm{S}_{1}$ states on the $\mathrm{Li}-\mathrm{H}$ internuclear distance.

\footnotetext{
$\ddagger^{\ddagger}$ The EKT-SSR-BH\&HLYP orbital energies are shifted to compensate for the difference between the calculated $(6.21 \mathrm{eV})$ and the experimental $(7.9 \mathrm{eV})$ first ionization potential of $\mathrm{LiH}$ at the ground state equilibrium geometry.
} 


\subsection{Ionization energies at conical intersection: PSB3}

It seems interesting to investigate variation of the ionization energies when passing through a conical intersection (CI); $\mathrm{CI}$ is a real crossing between the potential energy surfaces (PESs) of the electronic states with the same (space and spin) symmetry. ${ }^{22}$ During passage through CI a sudden change of the electronic states occurs. ${ }^{22}$ One of the best studied CIs is probably the one occurring between the $S_{1}$ and $S_{0}$ states of the 2,4-pentadieniminium cation, better known as PSB3 - a simple model of the retinal chromophore. ${ }^{81}$ In PSB3, a crossing between the $S_{1}$ and $S_{0}$ states occurs at approximately $90^{\circ}$ of torsion about the central double bond, see Fig. 6 . The $S_{1} / S_{0}$ crossing is lifted along the bond length alternation (BLA: difference between the average length of formally single bonds and of formally double bonds in the chain) coordinate; a standard path was optimized using the CASSCF method along the BLA coordinate. ${ }^{81}$

Using the geometries from Gozem et al. ${ }^{81}$ the $S_{1}$ and $S_{0}$ states of PSB3 were calculated along the BLA path using the SSR-BH\&HLYP/6-31G* method. In Fig. 6a, the results of the SSR calculations are compared with the results of the lattice regularized diffusion quantum Monte Carlo (LRDMC) calculations by Zen et al.. ${ }^{82}$ According to SSR-BH\&HLYP/6-31G* the $S_{1} / S_{0}$ crossing occurs at a BLA value of $0.056 \AA$ and at 68.6 $\mathrm{kcal} / \mathrm{mol}$ with respect to the ground state energy of the cis-PSB3 conformation. These values are in an excellent agreement with the LRDMC BLA value $0.062 \AA$ and the crossing energy $66.8 \mathrm{kcal} / \mathrm{mol}$.

Using the SSR analytic energy gradients and the gradient of the coupling term in Eq. (10) the branching plane (BP) vectors were calculated at the SSR crossing geometry. The BP vectors were orthogonalized using the Yarkony's formula ${ }^{83}$ and a loop with the diameter $0.01 \AA$ was set up around the crossing point. The $S_{1}-S_{0}$ energy difference round the 

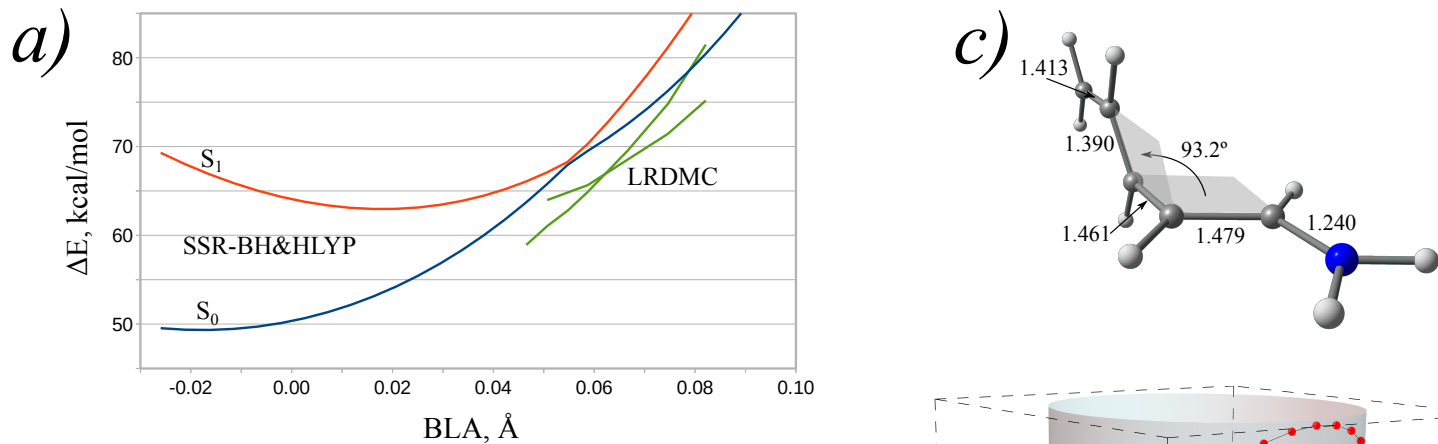

b)
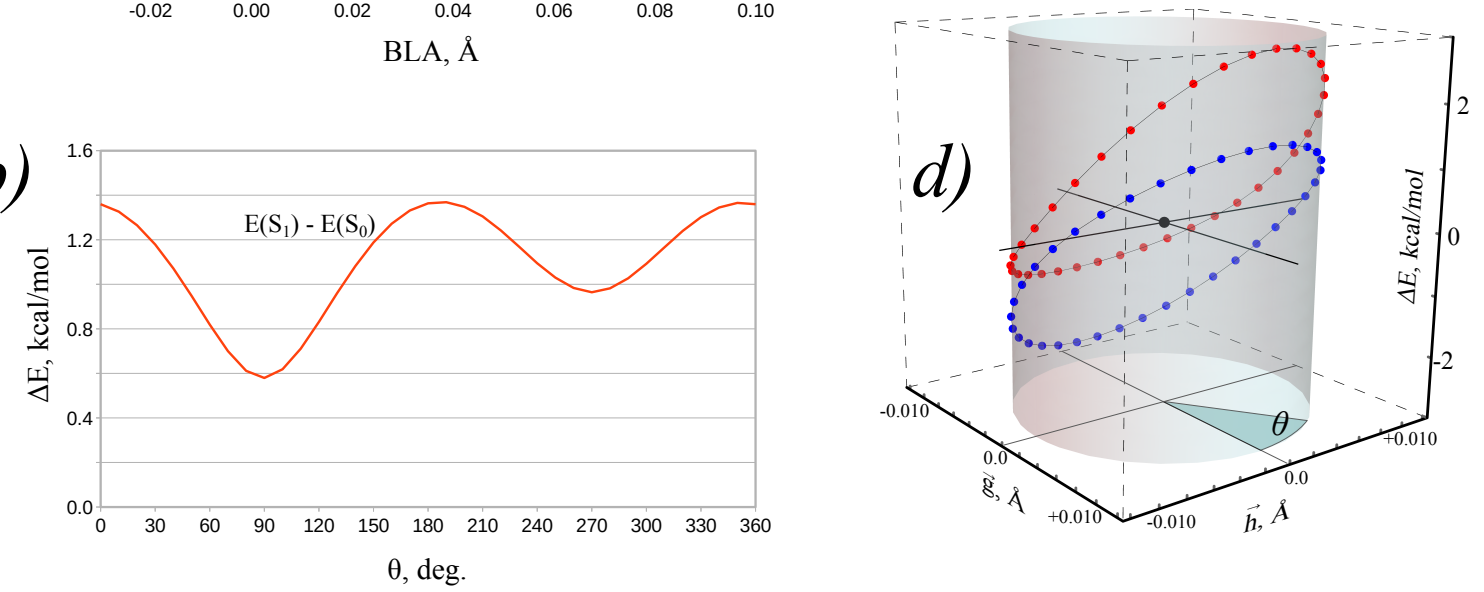

Figure 6: PSB3: Panel a): The relative energies (kcal/mol, with respect to the cis-PSB3 conformation) of the $S_{0}$ (blue curve) and the $S_{1}$ (red curve) states of PSB3 obtained with SSRBH\&HLYP / 6-311G ${ }^{* *}$ in comparison with the LRDMC 82 (green curves) energies along the BLA path. ${ }^{81}$ Panel $b$ ): The $S_{1}-S_{0}$ energy difference round a loop (radius $0.01 \AA$ ) within the branching plane around the $S_{1} / S_{0}$ crossing point at BLA $=0.056 \AA$. Panel c): The geometry of PSB3 at the $S_{1} / S_{0}$ crossing point. Panel $d$ ): A $3 D$ representation of the $S_{1}$ (red) and $S_{0}$ (blue) relative energies ( $\mathrm{kcal} / \mathrm{mol}$, with respect to the crossing point) round a loop in the branching plane.

loop is shown in Fig. $6 b$ and the $S_{1}$ and $S_{0}$ energies in Fig. $6 d$. As the $S_{1}$ and $S_{0}$ states do not cross when going round the loop (the energy difference is always positive), the crossing point qualifies as a CI. ${ }^{24}$

The $S_{1}$ and $S_{0}$ states in the vicinity of the CI in PSB3 can be described in terms of the diradical (DIR) and charge transfer (CT) electronic configurations. ${ }^{24}$ The former corresponds to homolytic breaking of the central $\pi$-bond of PSB3 and leaves the positive 

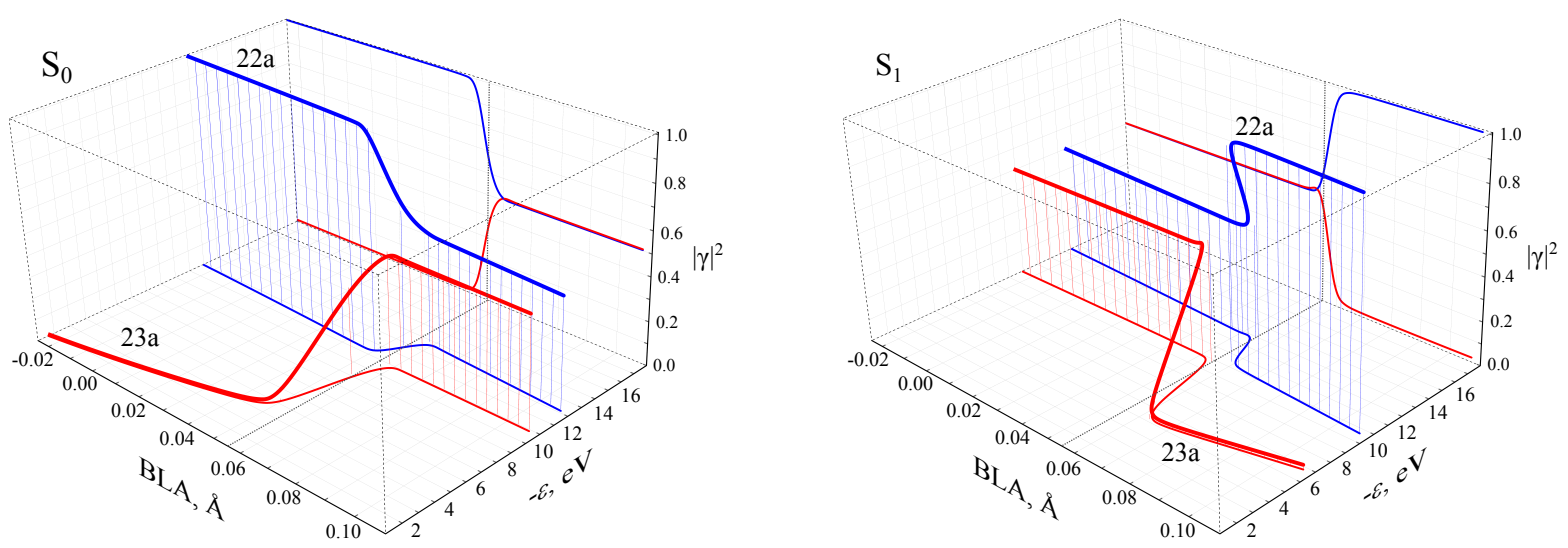

Figure 7: 3D plots showing the dependence of the energies and the norms of the $22 a$ and $23 a$ Dyson's orbitals of PSB3 in the $S_{0}$ state (left panel) and in the $S_{1}$ state (right panel) on the BLA parameter.

charge on the cationic nitrogen. The latter corresponds to heterolytic breaking of the central $\pi$-bond, which causes shifting of the positive charge from the nitrogen atom to the opposite end of the chain. ${ }^{24,81}$ As the DIR configuration is well approximated by an OSS-type wavefunction, it is expected that the frontier Dyson's orbitals (22a and 23a) should acquire fractional norms. The CT configuration corresponds to a closed-shell electronic structure well approximated by a single determinant; hence, the Dyson's orbital $22 a$ should have nearly unity norm and the $23 a^{\prime}$ s norm should be near zero.

The dependence of the energy and the norm of the 22a and 23a Dyson's orbitals of PSB3 on the magnitude of the BLA distortion is shown in Fig. 7. In the $\mathrm{S}_{0}$ state (the left panel), a rapid change from the CT configuration, characterized by the 1 and 0 norms, to the DIR configuration, characterized by equal norms of the frontier Dyson's orbitals, occurs, as the geometry passes the CI. An opposite picture is observed in the $S_{1}$ state, where the electronic structure rapidly changes from DIR to CT. According to the previous investigations of PSB3, ${ }^{24,81}$ this picture is to be expected. As the energies of the frontier 
Dyson's orbitals are sufficiently different (by ca. $2 \mathrm{eV}$ ), one may expect an occurrence (or a disappearance) of the respective peaks (e.g., single peak suddenly splits in two or vice versa) in TRPES measurements, when passage through a CI occurs.
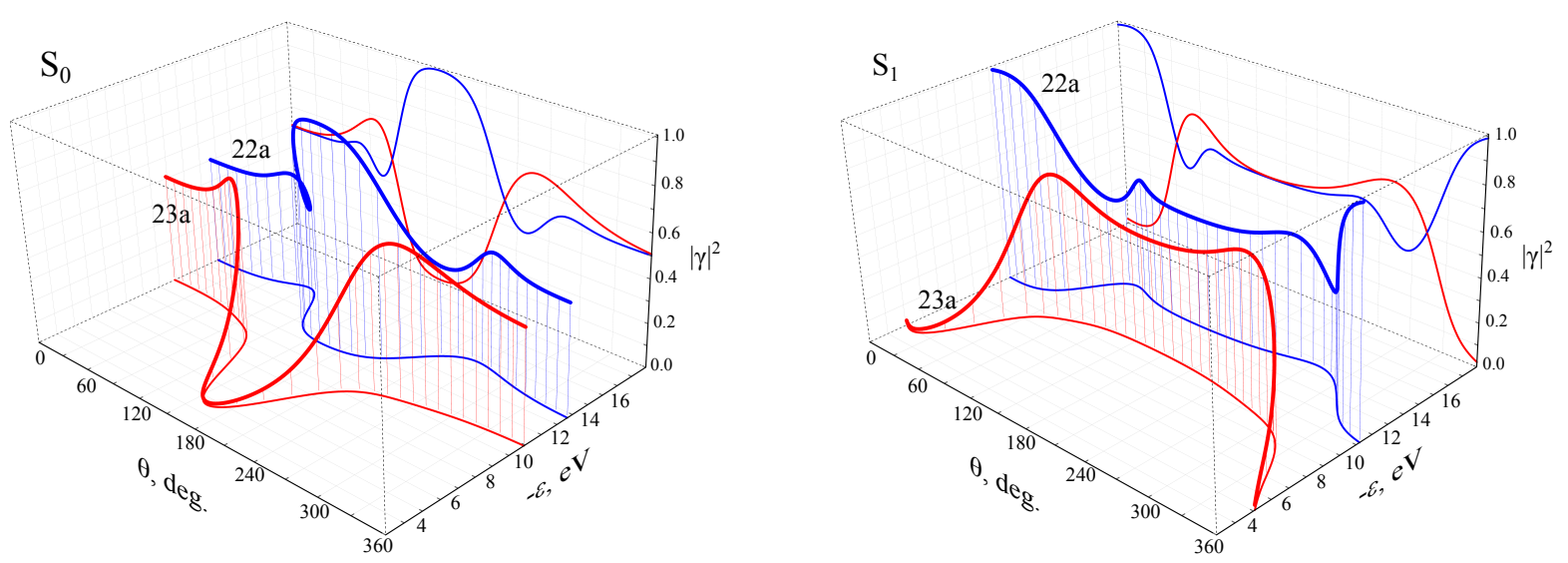

Figure 8: 3D plots showing the dependence of the energies and the norms of the $22 a$ and $23 a$ Dyson's orbitals of PSB3 in the $S_{0}$ state (left panel) and in the $S_{1}$ state (right panel) on the angle $\theta$ parameterizing the loop around the $S_{1} / S_{0}$ crossing point. See Fig. 6 for detail of the loop.

Plots of the energies and the norms of the frontier Dyson's orbitals round the loop in the branching plane of the CI in PSB3, see Fig. 8, reveals that a gradual variation of the $S_{0}$ and $S_{1}$ states between a closed-shell $C T$ and and open-shell DIR configurations takes place. Variation of the norms round the loop displays pattern similar to the one observed for dissociating $\mathrm{LiH}$. This picture seems to be typical for an avoided crossing, where a strong mixing between the states undergoing the crossing takes place. In the $\mathrm{S}_{0}$ state, the CT configuration dominates the electronic structure in the direction of the negative gradient difference vector (the vector $\mathbf{g}$ in Fig. 6) and the transition to the DIR configuration gradually occurs along the direction of the coupling gradient vector $(\mathbf{h}$ in Fig. 6). As the loop reaches the positive side of the $\mathbf{g}$ direction, the electronic structure of $S_{0}$ becomes completely diradical. An opposite picture holds in the $S_{1}$ electronic state 
of PSB3. This picture suggests that the splitting of the PES peak (or collapse of the two highest peaks) should occur even if a nuclear trajectory passes near a CI, in the region of an avoided crossing between the two electronic states.

\section{Conclusions}

In this work, an EKT-based ${ }^{36,39}$ computation of the ionization energies and the respective Dyson's orbitals was developed and implemented in connection with an ensemble DFT methodology, ${ }^{19-21}$ the SSR method. ${ }^{17,18,23,41,42}$ The new EKT-SSR methodology was tested in the computation of the ionization energies in the ground and excited states of several closed-shell molecules, as well as strongly correlated systems, such as ozone, dissociating $\mathrm{H}_{2}$ and $\mathrm{LiH}$ molecules, and the PSB3 cation. In the case of PSB3, the behavior of the ionization energies and the respective Dyson's orbitals was inspected in the region encompassing an $S_{1} / S_{0}$ conical intersection.

When the electronic state of the molecule is accurately approximated by a single determinant configuration, which is typical for the most of closed-shell molecules, the results of the EKT-SSR computations closely reproduce the ionization energies evaluated in the usual way by the Koopmans' theorem. ${ }^{4}$ For multi-reference electronic configurations, the occurrence of ionization states with fractionally normalized Dyson's orbitals was observed in the EKT-SSR computations. The fractional normalization of the Dyson's orbitals matches the fractional occupations of the frontier Kohn-Sham orbitals obtained in eDFT. ${ }^{43-45}$ As the norms of the Dyson's orbitals are connected with the probability of the respective ionization channels, ${ }^{3}$ the onset of multi-reference electronic structure, e.g., during photoreactions occurring in the excited states, can manifest itself in rapid varia- 
tions of the intensities of the time-resolved PES signals. For example, passage through the region of a conical intersection may result in splitting of the first ionization peak in the excited state in two peaks with smaller intensity.

The developed EKT-SSR method is very easy to implement and use; all the ingredients needed for the computation of the ionization energies are already present in the analytical energy gradient. Hence, on-the-fly computation of ionization energies during the nonadiabatic molecular dynamics simulations can be easily implemented and carried out at no additional cost. As the EKT-based computation can be also implemented for linearresponse DFT methods, such as, e.g., MRSF-TDDFT, 57 a variety of computational methods can be used to model TRPES signals simultaneously with the NAMD simulations.

Although this work is focused on the development of a practically accessible methodology, the merger of EKT with eDFT methods has a potential for deriving the exact theoretical approach to obtaining the ionization energies for ensembles of charge neutral excitations. It is to be noted that, besides charge neutral excitations, GOK-eDFT ${ }^{21}$ can be used in connection with charged excitations, such as in the N-centered eDFT methodology. ${ }^{33,34}$ In the latter case, the ionization energies can be obtained directly, from differentiation with respect to the ensemble weights. ${ }^{33,34}$ With an ensemble of the charge neutral excitations, as in the SSR method, differentiation with respect to the ensemble weights yields the excitation, rather than ionization, energies and the use of EKT offers a solution to the problem of obtaining the ionization energies. It is noteworthy that the use of an ensemble of neutral excitations in the SSR method does not violate the most significant requirements for the exact eDFT methodology, such as the presence of the derivative discontinuity (see the Appendix A) in the energies and properties. However, it offers a straightforward approach to obtaining the electronic excitations within a time- 
independent formalism; the excitations which possess the exact properties, e.g., with regard to proper description of the real and avoided crossings between the potential energy surfaces of the ground and excited states.

\section{Acknowledgement}

This work was supported by the the National Research Foundation of Korea (NRF) grant 2019H1D3A2A02102948.

\section{A Derivative discontinuity in EKT-SSR orbital energies}

We were asked by an anonymous reviewer to provide evidence that the SSR/REKS methodology correctly treats the derivative discontinuity of the XC energy. The derivative discontinuity ${ }^{20,35,84,85}$ manifests itself as a discontinuity of the derivative of the XC energy with respect to the total number of particles $\delta E_{X C} / \delta N$, when $N$ is passing an integer number.* This leads, inter alia, to a stepwise charge transfer between atoms (or fragments) in a molecule and to a step-like variation of the orbital energy when the total number of electrons becomes integer.

Although an evidence of the derivative discontinuity in the electron transfer described by the SSR method was presented already in Ref. 86, for the sake of argument, we demonstrate manifestations of the derivative discontinuity in the Dyson's orbitals energies obtained by the EKT-SSR method in this work. The SSR (and REKS) method is not defined for fractional total number of electrons. Hence, no direct calculation of the $\delta E_{X C} / \delta N$

${ }^{*}$ Note that, in $\mathrm{N}$-centered eDFT, the derivative discontinuity is related to the discontinuity of the derivative of the ensemble XC energy with respect to the ensemble weighting factor. ${ }^{33}$ 
Figure A1: Upper panel: Dependence on the Mulliken charge on an $\mathrm{H}$ atom in the stretched $\mathrm{H}_{2}$ molecule $(\mathrm{R}(\mathrm{H}-\mathrm{H})=4 \AA)$ on a uniform electric field applied along the molecular axis. Lower panel: Dependence of the energy of the Dyson's orbital localized predominantly on the right $\mathrm{H}$ atom on the Mulliken charge on that atom. Solid lines show the results of the SSR calculations (red: SSR-BLYP; blue: SSR-BHHLYP), dashed lines the results of the conventional RKS calculations (red: BLYP; blue: BHHLYP).

derivative is possible in the context of the method. Instead, a system of two nearly noninteracting atoms is used, as represented by the $\mathrm{H}_{2}$ molecule at $\mathrm{R}(\mathrm{H}-\mathrm{H})=4 \AA$ shown in 
Fig. A1. The total number of electrons in the molecule is conserved, however application of a uniform electric field along the molecular axis can drive the electron population from atom to atom. Such a model system can also be viewed as an indirect implementation of an ensemble of neutral $\left(\mathrm{H}^{\bullet}-\mathrm{H}^{\bullet}\right)$ and charged $\left(\mathrm{H}^{+}-\mathrm{H}^{-}\right.$and $\left.\mathrm{H}^{-}-\mathrm{H}^{+}\right)$states, which bears similarity to ensemble used in N-centered eDFT. ${ }^{33}$ Then, the electric field plays a role of an external perturbation that leads to variations of the weighting factors of the neutral and charged components of the ensemble.

When no field is present, the charge distribution is symmetric and both atoms are electrically neutral. The field breaks the symmetry of the charge distribution and drives the electronic density to one end of the molecule. If the derivative discontinuity is present in the $\mathrm{XC}$ energy, the electron transfer from atom to atom occurs in a stepwise manner, one electron after another. ${ }^{84,85}$ This is clearly seen in the upper panel of Fig. A1 where the Mulliken charge on an $\mathrm{H}$ atom in the stretched $\mathrm{H}_{2}$ is shown as obtained in the SSR-BLYP /6-311G ${ }^{* *}$ and the SSR-BH\&HLYP / 6-311G** calculations. For comparison, the Mulliken charges obtained by the conventional RKS methodology (with BLYP and BH\&HLYP) are shown in Fig. A1. The latter charges vary continuously with the field strength; thus showing the absence of the derivative discontinuity in the conventional approximate XC functionals. It is noteworthy that, even with a pure GGA functional, such as BLYP, the SSR method correctly describes stepwise charge transfer. Similar results were obtained earlier in Ref. 86 for a donor-acceptor dyad.

The lower panel of Fig. A1 shows the dependence of the Dyson's orbital energy on the atomic Mulliken charge. The Dyson's orbital localized predominantly on one of the $\mathrm{H}$ atoms (the right atom in Fig. A1) is picked up. Naturally, when no field is present the two frontier Dyson's orbitals of $\mathrm{H}_{2}$ are delocalized over both atoms and have the same 
energies, see Fig. 2. Application of the field leads to fractional atomic population and the energy of the respective Dyson's orbital undergoes a step-like change, when passing through the integer atomic population.

Note that the orbital energies are sensitive to the interaction with the applied electric field. Hence, the energies in Fig. A1 are shown for very small atomic charges only, where the energies remain mostly constant. The step-like behavior of the Dyson's orbital energy is visible even for a pure GGA functional (BLYP), when used in connection with the SSR method. The conventional RKS methodology does not yield a step when the BLYP functional is used. The RKS BH\&HLYP method yields a small step as a consequence of the exact exchange energy included in the hybrid XC functional. However, the magnitude of the step is tiny as compared to the one produced by the SSR method. These results demonstrate the presence of the derivative discontinuity in the SSR energies and properties.

\section{References}

(1) Haight, R.; Bokor, J.; Stark, J.; Storz, R. H.; Freeman, R. R.; Bucksbaum, P. H. Picosecond Time-Resolved Photoemission Study of the $\operatorname{InP}(110)$ Surface. Phys. Rev. Lett. 1985, 54, 1302-1305.

(2) (a) Neumark, D. M. Time-Resolved Photoelectron Spectroscopy Of Molecules And Clusters. Annu. Rev. Phys. Chem. 2001, 52, 255-277; (b) Stolow, A. Femtosecond TimeResolved Photoelectron Spectroscopy Of Polyatomic Molecules. Annu. Rev. Phys. Chem. 2003, 54, 89-119; (c) Stolow, A.; Bragg, A. E.; Neumark, D. M. Femtosecond Time-Resolved Photoelectron Spectroscopy. Chem. Rev. 2004, 104, 1719-1758. 
(3) Spanner, M.; Patchkovskii, S.; Zhou, C.; Matsika, S.; Kotur, M.; Weinacht, T. C. Dyson norms in XUV and strong-field ionization of polyatomics: Cytosine and uracil. Phys. Rev. A 2012, 86, 053406.

(4) Koopmans, T. Über die Zuordnung von Wellenfunktionen und Eigenwerten zu den Einzelnen Elektronen Eines Atoms. Physica 1934, 1, 104-113.

(5) Bagus, P. S. Self-Consistent-Field Wave Functions for Hole States of Some Ne-Like and Ar-Like Ions. Phys. Rev. 1965, 139, A619-A634.

(6) Linderberg, J.; Öhrn, Y. Propagators in Quantum Chemistry; Academic Press: New York, 1973.

(7) Purvis, G. D.; Öhrn, Y. Accurate molecular ionization potentials from the electron propagator. Int. J. Quantum Chem. 1977, 12, 359-363.

(8) Ortiz, J. V. Electron propagator theory: an approach to prediction and interpretation in quantum chemistry. WIREs Comp. Mol. Sci. 2013, 3, 123-142.

(9) Hedin, L. New Method for Calculating the One-Particle Green's Function with Application to the Electron-Gas Problem. Phys. Rev. 1965, 139, A796-A823.

(10) Shirley, E. L.; Martin, R. M. GW quasiparticle calculations in atoms. Phys. Rev. B 1993, $47,15404-15412$.

(11) Golze, D.; Dvorak, M.; Rinke, P. The GW Compendium: A Practical Guide to Theoretical Photoemission Spectroscopy. Front. Chem. 2019, 7, 377.

(12) Pickup, B. T. On the theory of fast photoionization processes. Chem. Phys. 1977, 19, $193-208$. 
(13) Öhrn, Y.; Born, G. In Molecular Electron Propagator Theory and Calculations; Löwdin, P.O., Ed.; Advances in Quantum Chemistry; Academic Press, 1981; Vol. 13; pp 1 - 88.

(14) Ortiz, J. V. Brueckner orbitals, Dyson orbitals, and correlation potentials. Int. J. Quantum Chem. 2004, 100, 1131-1135.

(15) Melania Oana, C.; Krylov, A. I. Dyson orbitals for ionization from the ground and electronically excited states within equation-of-motion coupled-cluster formalism: Theory, implementation, and examples. J. Chem. Phys. 2007, 127, 234106.

(16) Thompson, A. L.; Martínez, T. J. Time-resolved photoelectron spectroscopy from first principles: Excited state dynamics of benzene. Faraday Discuss. 2011, 150, 293-311.

(17) Filatov, M. Spin-restricted ensemble-referenced Kohn-Sham method: basic principles and application to strongly correlated ground and excited states of molecules. WIREs Comput. Mol. Sci. 2015, 5, 146-167.

(18) Filatov, M. In Density-functional methods for excited states; Ferré, N., Filatov, M., HuixRotllant, M., Eds.; Top. Curr. Chem.; Springer: Heidelberg, 2016; Vol. 368; pp 97-124.

(19) (a) Valone, S. M. A one-to-one mapping between one-particle densities and some n-particle ensembles. J. Chem. Phys. 1980, 73, 4653-4655; (b) Lieb, E. H. Density functionals for Coulomb systems. Int. J. Quantum Chem. 1983, 24, 243-277; (c) Englisch, H.; Englisch, R. Hohenberg-Kohn Theorem and Non-V-Representable Densities. Physica 1983, A121, 253-268; (d) Englisch, H.; Englisch, R. Exact Density Functionals for Ground-State Energies. I. General Results. Phys. Stat. Sol. (b) 1984, 123, 711-721; (e) Englisch, H.; Englisch, R. Exact Density Functionals for Ground-State Energies II. Details and Remarks. Phys. Stat. Sol. (b) 1984, 124, 373-379. 
(20) Perdew, J. P.; Parr, R. G.; Levy, M.; Balduz Jr., J. L. Density-Functional Theory for Fractional Particle Number: Derivative Discontinuities of the Energy. Phys. Rev. Lett. $1982,49,1691-1694$.

(21) (a) Gross, E. K. U.; Oliveira, L. N.; Kohn, W. Rayleigh-Ritz variational principle for ensembles of fractionally occupied states. Phys. Rev. A 1988, 37, 2805-2808; (b) Gross, E. K. U.; Oliveira, L. N.; Kohn, W. Density-functional theory for ensembles of fractionally occupied states. I. Basic formalism. Phys. Rev. A 1988, 37, 2809-2820; (c) Oliveira, L. N.; Gross, E. K. U.; Kohn, W. Density-functional theory for ensembles of fractionally occupied states. II. Application to the He atom. Phys. Rev. A 1988, 37, 2821-2833; (d) Oliveira, L. N.; Gross, E. K. U.; Kohn, W. Ensemble-Density Functional Theory. Int. J. Quantum Chem.: Quantum Chem. Symp. 1990, 24, 707-716.

(22) (a) Atchity, G. J.; Xantheas, S. S.; Ruedenberg, K. Potential energy surfaces near intersections. J. Chem. Phys. 1991, 95, 1862-1876; (b) Yarkony, D. R. Diabolical Conical Intersections. Rev. Mod. Phys. 1996, 68, 985-1013; (c) Bernardi, F.; Olivucci, M.; Robb, M. A. Potential energy surface crossings in organic photochemistry. Chem. Soc. Rev. 1996, 25, 321-328.

(23) Filatov, M. Assessment of density functional methods for obtaining geometries at conical intersections in organic molecules. J. Chem. Theory Comput. 2013, 9, 4526-4541.

(24) Gozem, S.; Melaccio, F.; Valentini, A.; Filatov, M.; Huix-Rotllant, M.; Ferré, N.; Frutos, L. M.; Angeli, C.; Krylov, A. I.; Granovsky, A. A.; Lindh, R.; Olivucci, M. Shape of Multireference, Equation-of-Motion Coupled-Cluster, and Density Functional Theory Potential Energy Surfaces at a Conical Intersection. J. Chem. Theory Comput. 2014, 10, 3074-3084. 
(25) Nikiforov, A.; Gamez, J. A.; Thiel, W.; Huix-Rotllant, M.; Filatov, M. Assessment of approximate computational methods for conical intersections and branching plane vectors in organic molecules. J. Chem. Phys. 2014, 141, 124122.

(26) Filatov, M.; Min, S. K.; Kim, K. S. Direct Nonadiabatic Dynamics by Mixed QuantumClassical Formalism Connected with Ensemble Density Functional Theory Method: Application to trans-Penta-2,4-dieniminium Cation. J. Chem. Theory Comput. 2018, 14, 4499-4512.

(27) Filatov, M.; Paolino, M.; Min, S. K.; Kim, K. S. Fulgides as Light-Driven Molecular Rotary Motors: Computational Design of a Prototype Compound. J. Phys. Chem. Lett. 2018, 9, 4995-5001.

(28) Filatov, M.; Min, S. K.; Kim, K. S. Non-adiabatic dynamics of ring opening in cyclohexa-1,3-diene described by an ensemble density-functional theory method. Mol. Phys. 2019, 117, 1128-1141.

(29) Filatov, M.; Min, S. K.; Choi, C. H. Theoretical Modelling of the Dynamics of Primary Photoprocess of Cyclopropanone. Phys. Chem. Chem. Phys. 2019, 21, 2489-2498.

(30) Filatov, M.; Paolino, M.; Min, S. K.; Choi, C. H. Design and photoisomerization dynamics of a new family of synthetic 2-stroke light driven molecular rotary motors. Chem. Commun. 2019, 55, 5247-5250.

(31) Liang, R.; Liu, F.; Martínez, T. J. Nonadiabatic Photodynamics of Retinal Protonated Schiff Base in Channelrhodopsin 2. J. Phys. Chem. Lett. 2019, 10, 2862-2868.

(32) Yu, J. K.; Liang, R.; Liu, F.; Martínez, T. J. First-Principles Characterization of the 
Elusive I Fluorescent State and the Structural Evolution of Retinal Protonated Schiff Base in Bacteriorhodopsin. J. Am. Chem. Soc. 2019, 141, 18193-18203.

(33) Senjean, B.; Fromager, E. Unified formulation of fundamental and optical gap problems in density-functional theory for ensembles. Phys. Rev. A 2018, 98, 022513.

(34) Senjean, B.; Fromager, E. N-centered ensemble density-functional theory for open systems. Int. J. Quantum Chem. n/a, e26190.

(35) Perdew, J. P.; Levy, M. Physical Content of the Exact Kohn-Sham Orbital Energies: Band Gaps and Derivative Discontinuities. Phys. Rev. Lett. 1983, 51, 1884-1887.

(36) (a) Morrell, M. M.; Parr, R. G.; Levy, M. Calculation of ionization potentials from density matrices and natural functions, and the long-range behavior of natural orbitals and electron density. J. Chem. Phys. 1975, 62, 549-554; (b) Smith, D. W.; Day, O. W. Extension of Koopmans' theorem. I. Derivation. J. Chem. Phys. 1975, 62, 113-114.

(37) Katriel, J.; Davidson, E. R. Asymptotic behavior of atomic and molecular wave functions. Proc. Nat. Acad. Sci. 1980, 77, 4403-4406.

(38) Vanfleteren, D.; Van Neck, D.; Ayers, P. W.; Morrison, R. C.; Bultinck, P. Exact ionization potentials from wavefunction asymptotics: The extended Koopmans' theorem, revisited. J. Chem. Phys. 2009, 130, 194104.

(39) Cioslowski, J.; Piskorz, P.; Liu, G. Ionization potentials and electron affinities from the extended Koopmans' theorem applied to energy-derivative density matrices: The EKTMPn and EKTQCISD methods. J. Chem. Phys. 1997, 107, 6804-6811. 
(40) Filatov, M.; Liu, F.; Martínez, T. J. Analytical derivatives of the individual state energies in ensemble density functional theory method. I. General formalism. J. Chem. Phys. 2017, 147, 034113.

(41) (a) Filatov, M.; Shaik, S. A spin-restricted ensemble-referenced Kohn-Sham method and its application to diradicaloid situations. Chem. Phys. Lett. 1999, 304, 429-437; (b) Moreira, I. d. P. R.; Costa, R.; Filatov, M.; Illas, F. Restricted ensemble-referenced Kohn-Sham versus broken symmetry approaches in density functional theory: Magnetic coupling in $\mathrm{Cu}$ binuclear complexes. J. Chem. Theory Comput. 2007, 3, 764-774.

(42) Kazaryan, A.; Heuver, J.; Filatov, M. Excitation Energies from Spin-Restricted Ensemble-Referenced Kohn-Sham Method: A State-Average Approach $\dagger$. J. Phys. Chem. A 2008, 112, 12980-12988.

(43) Schipper, P. R. T.; Gritsenko, O. V.; Baerends, E.-J. One-determinantal pure state versus ensemble Kohn-Sham solutions in the case of strong electron correlation: $\mathrm{CH} 2$ and C2. Theor. Chem. Acc. 1998, 99, 329-343.

(44) Schipper, P. R. T.; Gritsenko, O. V.; Baerends, E.-J. Benchmark calculations of chemical reactions in density functional theory: Comparison of the accurate Kohn-Sham solution with generalized gradient approximations for the $\mathrm{H} 2+\mathrm{H}$ and $\mathrm{H} 2+\mathrm{H} 2$ reactions. J. Chem. Phys. 1999, 111, 4056-4067.

(45) Morrison, R. C. Electron correlation and noninteracting v-representability in density functional theory: The Be isoelectronic series. J. Chem. Phys. 2002, 117, 10506-10511.

(46) Filatov, M.; Martínez, T. J.; Kim, K. S. Using the GVB Ansatz to develop ensemble 
DFT method for describing multiple strongly correlated electron pairs. Phys. Chem. Chem. Phys. 2016, 18, 21040-21050.

(47) Filatov, M.; Liu, F.; Kim, K. S.; Martínez, T. J. Self-consistent implementation of ensemble density functional theory method for multiple strongly correlated electron pairs. J. Chem. Phys. 2016, 145, 244104.

(48) Filatov, M.; Martínez, T. J.; Kim, K. S. Description of ground and excited electronic states by ensemble density functional method with extended active space. J. Chem. Phys. 2017, 147, 064104.

(49) Deur, K.; Fromager, E. Ground and excited energy levels can be extracted exactly from a single ensemble density-functional theory calculation. J. Chem. Phys. 2019, 150, 094106.

(50) Hirao, K.; Nakatsuji, H. General SCF operator satisfying correct variational condition. J. Chem. Phys. 1973, 59, 1457-1462.

(51) Perera, S. A.; Watts, J. D.; Bartlett, R. J. A theoretical study of hyperfine coupling constants. J. Chem. Phys. 1994, 100, 1425-1434.

(52) Pulay, P. Ab initio calculation of force constants and equilibrium geometries in polyatomic molecules. Mol. Phys. 1969, 17, 197-204.

(53) Stålring, J.; Bernhardsson, A.; Lindh, R. Analytical gradients of a state average MCSCF state and a state average diagnostic. Mol. Phys. 2001, 99, 103-114.

(54) Peters, G.; Wilkinson, J. H. Ax $=\lambda$ Bx and the Generalized Eigenproblem. SIAM J. Num. Analysis 1970, 7, 479-492. 
(55) Giesbertz, K. J. H.; van Leeuwen, R. Natural occupation numbers: When do they vanish? J. Chem. Phys. 2013, 139, 104109.

(56) Kent, P. R. C.; Hood, R. Q.; Towler, M. D.; Needs, R. J.; Rajagopal, G. Quantum Monte Carlo calculations of the one-body density matrix and excitation energies of silicon. Phys. Rev. B 1998, 57, 15293-15302.

(57) Lee, S.; Filatov, M.; Lee, S.; Choi, C. H. Eliminating spin-contamination of spin-flip time dependent density functional theory within linear response formalism by the use of zeroth-order mixed-reference (MR) reduced density matrix. J. Chem. Phys. 2018, 149, 104101.

(58) (a) Schmidt, M. W.; Baldridge, K. K.; Boatz, J. A.; Elbert, S. T.; Gordon, M. S.; Jensen, J. J.; Koseki, S.; Matsunaga, N.; Nguyen, K. A.; Su, S.; Windus, T. L.; Dupuis, M.; Montgomery, J. A. J. Comput. Chem. 1993, 14, 1347-1363; (b) Gordon, M.; Schmidt, M. In Theory and Applications of Computational Chemistry, the first forty years; Dykstra, C. E., Frenking, G., Kim, K. S., Scuseria, G. E., Eds.; Elsevier: Amsterdam, 2005; pp 1167-1189.

(59) Krishnan, R.; Binkley, J. S.; Seeger, R.; Pople, J. A. Self-consistent molecular orbital methods. XX. A basis set for correlated wave functions. J. Chem. Phys. 1980, 72, 650654.

(60) (a) Becke, A. D. A New Mixing of Hartree-Fock and Local Density-Functional Theories. J. Chem. Phys. 1993, 98, 1372-1377; (b) Becke, A. D. Density-Functional Exchange-Energy Approximation with Correct Asymptotic Behavior. Phys. Rev. A 1988, 38, 3098-3100; (c) Lee, C.; Yang, W.; Parr, R. G. Development of the Colle- 
Salvetti Correlation-Energy Formula into a Functional of the Electron Density. Phys. Rev. B 1988, 37, 785-789.

(61) Hay, P. J.; Dunning, T. H.; Goddard, W. A. Configuration interaction studies of O3 and O+3. Ground and excited states. J. Chem. Phys. 1975, 62, 3912-3924.

(62) Takeshita, T. Y.; Lindquist, B. A.; Dunning, T. H. Insights into the Electronic Structure of Ozone and Sulfur Dioxide from Generalized Valence Bond Theory: Bonding in O3 and SO2. J. Phys. Chem. A 2015, 119, 7683-7694.

(63) Miliordos, E.; Xantheas, S. S. On the Bonding Nature of Ozone (O3) and Its SulfurSubstituted Analogues SO2, OS2, and S3: Correlation between Their Biradical Character and Molecular Properties. J. Am. Chem. Soc. 2014, 136, 2808-2817.

(64) Kotur, M.; Weinacht, T. C.; Zhou, C.; Matsika, S. Strong-Field Molecular Ionization from Multiple Orbitals. Phys. Rev. X 2011, 1, 021010.

(65) Chong, D. P.; Gritsenko, O. V.; Baerends, E. J. Interpretation of the Kohn-Sham orbital energies as approximate vertical ionization potentials. J. Chem. Phys. 2002, 116, 17601772.

(66) Handy, N. C.; Marron, M. T.; Silverstone, H. J. Long-Range Behavior of Hartree-Fock Orbitals. Phys. Rev. 1969, 180, 45-48.

(67) Politzer, P.; Abu-Awwad, F. A comparative analysis of Hartree-Fock and Kohn-Sham orbital energies. Theor. Chem. Acc. 1998, 99, 83-87.

(68) NIST Standard Reference Database Number 101. Release 20. 2019; http: //cccbdb. nist.gov/. 
(69) Ranasinghe, D. S.; Margraf, J. T.; Perera, A.; Bartlett, R. J. Vertical valence ionization potential benchmarks from equation-of-motion coupled cluster theory and QTP functionals. J. Chem. Phys. 2019, 150, 074108.

(70) Cederbaum, L. S.; Domcke, W.; von Niessen, W.; Kraemer, W. A difficult assignment problem. The ionic states of ozone and sulphur dioxide. Mol. Phys. 1977, 34, 381-396.

(71) Barysz, M.; Ritby, M.; Bartlett, R. J. Fock space multi-reference coupled-cluster study of excitation energies and dipole oscillator strengths of ozone. Chem. Phys. Lett. 1992, 193, 373-379.

(72) Ortiz, J. V. Single-reference electron propagator calculations on vertical ionization energies of ozone. Chem. Phys. Lett. 1998, 297, 193-199.

(73) McKellar, A. J.; Yeager, D. L.; Nichols, J. A.; Golab, J. T. Low-lying ionization potentials of $\mathrm{O} 3$ and $\mathrm{NO} 2$ using the multiconfigurationa lspin tensor electron propagator method. J. Chem. Phys. 1996, 105, 9927-9932.

(74) McKellar, A. J.; Heryadi, D.; Yeager, D. L.; Nichols, J. A. Complete basis set limit ionization potentials of $\mathrm{O} 3$ and $\mathrm{NO} 2$ using the multiconfigurational spin tensor electron propagator method (MCSTEP). Chem. Phys. 1998, 238, 1-9.

(75) Ohtsuka, Y.; y. Hasegawa, J.; Nakatsuji, H. Excited and ionized states of ozone studied by the MEG (multi-exponentially generated)/EX (excited)-MEG method. Chem. Phys. 2007, 332, 262-270.

(76) Katsumata, S.; Shiromaru, H.; Kimura, T. Photoelectron Angular Distribution and Assignment of Photoelectron Spectrum of Ozone. Bull. Chem. Soc. Japan 1984, 57, 1784-1788. 
(77) Loos, P.-F.; Scemama, A.; Blondel, A.; Garniron, Y.; Caffarel, M.; Jacquemin, D. A Mountaineering Strategy to Excited States: Highly Accurate Reference Energies and Benchmarks. J. Chem. Theory Comput. 2018, 14, 4360-4379.

(78) Loos, P.-F.; Lipparini, F.; Boggio-Pasqua, M.; Scemama, A.; Jacquemin, D. A Mountaineering Strategy to Excited States: Highly Accurate Energies and Benchmarks for Medium Sized Molecules. J. Chem. Theory Comput. 2020, 0, 0.

(79) Aryasetiawan, F.,; Gunnarsson, O.,; Rubio, A., Excitation energies from timedependent density-functional formalism for small systems. Europhys. Lett. 2002, 57, 683-689.

(80) Coulson, C. A.; Fischer, I. XXXIV. Notes on the molecular orbital treatment of the hydrogen molecule. Phil. Mag. 1949, 40, 386-393.

(81) Gozem, S.; Huntress, M.; Schapiro, I.; Lindh, R.; Granovsky, A. A.; Angeli, C.; Olivucci, M. Dynamic Electron Correlation Effects on the Ground State Potential Energy Surface of a Retinal Chromophore Model. J. Chem. Theory Comput. 2012, 8, 4069-4080.

(82) Zen, A.; Coccia, E.; Gozem, S.; Olivucci, M.; Guidoni, L. Quantum Monte Carlo Treatment of the Charge Transfer and Diradical Electronic Character in a Retinal Chromophore Minimal Model. J. Chem. Theory Comput. 2015, 11, 992-1005.

(83) Yarkony, D. R. On the adiabatic to diabatic states transformation near intersections of conical intersections. J. Chem. Phys. 2000, 112, 2111-2120.

(84) Perdew, J. P. In Density Functional Methods In Physics; Dreizler, R. M., da Providência, J., Eds.; Springer US: Boston, MA, 1985; pp 265-308. 
(85) Perdew, J. P. In Density Functional Theory of Many-Fermion Systems; Löwdin, P.-O., Ed.; Adv. Quantum Chem.; Academic Press, 1990; Vol. 21; pp 113 - 134.

(86) Filatov, M. Description of electron transfer in the ground and excited states of organic donor-acceptor systems by single-reference and multi-reference density functional methods. J. Chem. Phys. 2014, 141, 124123. 


\section{Graphical TOC Entry}

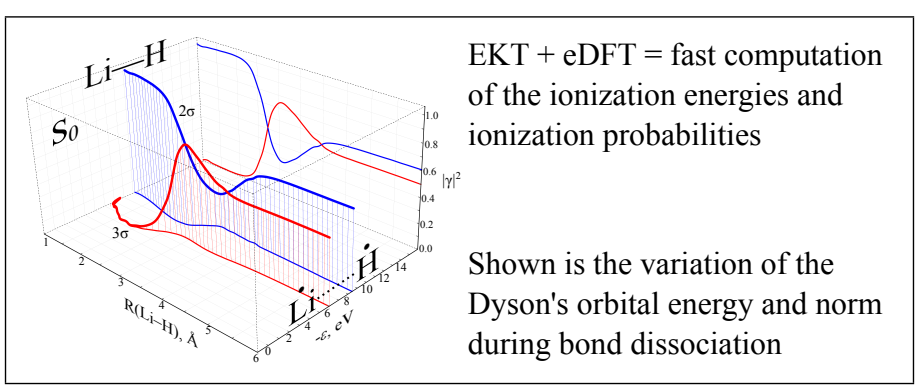

